\title{
Monitoring the training dose and acute fatigue response during elbow flexor resistance training using a custom-made resistance band
}

\author{
Jingjing Yang ${ }^{1}$, Hongbin Xu ${ }^{2}$, Juke Liang ${ }^{2}$, Jongyeob Jeong ${ }^{3}$, Taojin Xu ${ }^{\text {Corresp. } 2,3}$ \\ ${ }^{1}$ Faculty of Civil Aviation and Aeroautics, Kunming University of Science and Technology, Kunming, China \\ 2 College of Mechanical Engineering, Chongqing University of Technology, Chongqing, China \\ ${ }^{3}$ Graduate School of Sciences and Technology for Innovation, Yamaguchi University, Ube, Japan \\ Corresponding Author: Taojin Xu \\ Email address: w503wc@yamaguchi-u.ac.jp
}

Background: Home-based resistance training offers an alternative to traditional, hospital-based or rehabilitation center-based resistance training, and has attracted much attention recently. However, without the supervision of a therapist or the assistance of an exercise monitoring system, one of the biggest challenges of home-based resistance training is that the therapist may not know if the patient has performed the exercise as prescribed. A lack of objective measurements limits the ability of researchers to evaluate the outcome of exercise interventions and choose suitable training doses.

Objective: To create an automated and objective method for segmenting resistance force data into contraction phase-specific segments and calculate the repetition number and time-under-tension (TUT) during elbow flexor resistance training. A pilot study was conducted to evaluate the performance of the segmentation algorithm and to show the capability of the system in monitoring the compliance of patients to a prescribed training program in a practical resistance training setting.

Methods: Six subjects ( 3 male and 3 female) volunteered to participate in a fatigue and recovery experiment (5-minutes intermittent submaximal contraction (ISC); 1-minute rest; 2-minutes ISC). A custom-made resistance band was used to help subjects perform biceps curl resistance exercises and the resistance was recorded through a load cell. The maximum and minimum values of the force-derivative were obtained as distinguishing features, and a segmentation algorithm was proposed to divide the biceps curl cycle into concentric (CON), eccentric (ECC), and isometric (ISOM) contraction, and rest phases. Two assessors, who were unfamiliar with the study, were recruited to manually pick the visually observed cut-off point between two contraction phases, and the TUT was calculated and compared to evaluate performance of the segmentation algorithm.

Results: The segmentation algorithm was programmatically implemented and the repetition number and contraction-phase specific TUT were calculated. During ISOM, the average TUT (3.75 $\pm 0.62 \mathrm{~s}$ ) was longer than the prescribed 3 seconds, indicating that most subjects did not perform the exercise as prescribed. There was a good TUT agreement and contraction segment agreement between the proposed algorithm and the assessors.

Conclusion: The good agreement in TUT between the proposed algorithm and the assessors indicates that the proposed algorithm can correctly segment the contraction into contraction phase-specific parts, thereby providing clinicians and researchers with an automated and objective method for quantifying home-based elbow flexor resistance training. The instrument is easy to use and cheap, and the 
segmentation algorithm is programmatically implemented, indicating good application prospect of the method in a practical setting. 
2 Monitoring the training dose and acute fatigue response during elbow flexor 3 resistance training using a custom-made resistance band

5 Jingjing Yang ${ }^{1}$, Hongbin $\mathrm{Xu}^{2}$, Juke Liang ${ }^{2}$, Jongyeob Jeong ${ }^{3}$, Taojin $\mathrm{Xu}^{2,3}$

$7{ }^{1}$ Faculty of Civil Aviation and Aeroautics, Kunming University of Science and Technology,

8 Kunming, China

$9 \quad{ }^{2}$ College of Mechanical Engineering, Chongqing University of Technology, Chongqing, China

$10{ }^{3}$ Graduate School of Sciences and Technology for Innovation, Yamaguchi University, Ube, 11 Japan

Corresponding Author:

14 Taojin $\mathrm{Xu}^{2,3}$

15 Graduate School of Science and Engineering, Yamaguchi University, 2-16-1 Tokiwadai, Ube, 16 755-8611, Japan

17 Email address: w503wc@yamaguchi-u.ac.jp

18 Tel.: (+81) 0707-483-2299

\section{Abstract}

Background: Home-based resistance training offers an alternative to traditional, hospital-based or rehabilitation center-based resistance training, and has attracted much attention recently. However, without the supervision of a therapist or the assistance of an exercise monitoring system, one of the biggest challenges of home-based resistance training is that the therapist may not know if the patient has performed the exercise as prescribed. A lack of objective measurements limits the ability of researchers to evaluate the outcome of exercise interventions and choose suitable training doses.

Objective: To create an automated and objective method for segmenting resistance force data 
30

31

32

33

34

35

36

37

38

39

40

41

42

43

44

45

46

47

48

49

50

51

52

53

54

55

56

57

58

59

60

61

62

63

tension (TUT) during elbow flexor resistance training. A pilot study was conducted to evaluate the performance of the segmentation algorithm and to show the capability of the system in monitoring the compliance of patients to a prescribed training program in a practical resistance training setting.

Methods: Six subjects (3 male and 3 female) volunteered to participate in a fatigue and recovery experiment (5-minutes intermittent submaximal contraction (ISC); 1-minute rest; 2-minutes ISC). A custom-made resistance band was used to help subjects perform biceps curl resistance exercises and the resistance was recorded through a load cell. The maximum and minimum values of the force-derivative were obtained as distinguishing features, and a segmentation algorithm was proposed to divide the biceps curl cycle into concentric (CON), eccentric (ECC), and isometric (ISOM) contraction, and rest phases. Two assessors, who were unfamiliar with the study, were recruited to manually pick the visually observed cut-off point between two contraction phases, and the TUT was calculated and compared to evaluate performance of the segmentation algorithm.

Results: The segmentation algorithm was programmatically implemented and the repetition number and contraction-phase specific TUT were calculated. During ISOM, the average TUT ( $3.75 \pm 0.62 \mathrm{~s})$ was longer than the prescribed 3 seconds, indicating that most subjects did not perform the exercise as prescribed. There was a good TUT agreement and contraction segment agreement between the proposed algorithm and the assessors.

Conclusion: The good agreement in TUT between the proposed algorithm and the assessors indicates that the proposed algorithm can correctly segment the contraction into contraction phase-specific parts, thereby providing clinicians and researchers with an automated and objective method for quantifying home-based elbow flexor resistance training. The instrument is easy to use and cheap, and the segmentation algorithm is programmatically implemented, indicating good application prospect of the method in a practical setting.

Keywords: Resistance Training; Home-based; Resistance Band; Elbow Flexor; Time-undertension

\section{Introduction}

Aging and illness can cause muscle wasting and weakness, eventually limiting the physical function of limbs [1]. Resistance training is an effective physical rehabilitation method used to improve muscle strength and physical function in elderly and patients with disability, 
64 recommended by national health organizations, such as the American College of Sports

65 Medicine and the American Heart Association [2,3]. The population in need of physical

66 rehabilitation is constantly increasing, however individuals, especially older adults and those

67 living in rural areas [4,5], lack easy access to public rehabilitation centers, because of financial or

68 physical constraints or limited therapist availability $[4,6]$. Even though the increase in the

69 number of rehabilitation therapists has outpaced the population growth in recent years [7], there

70 is still a shortage of trained rehabilitation service providers in aging societies.

71

72

73

74

75

76

77

78

79

80

81

82

83

84

85

86

87

88

89

90

91

92

93

94

95

96

97

98

99

100

101

\section{1}

Home-based resistance training offers an alternative to traditional, hospital-based or rehabilitation center-based resistance training, and has attracted much attention recently $[5,8]$. After the physiotherapist provides the patient with initial instructions on how to perform the exercise, home-based trainees need to complete the entire training program on their own. Without the supervision of a therapist or help of an exercise monitoring system, and considering that the training is a long process that lasts weeks or months, one of the biggest challenges in home-based resistance training is that subjects do not follow their exercise prescription $[9,10,11]$. In particular, it has been shown that patients perform the exercises either too fast or too slow, resulting in too short or too long contraction time with too few or too many repetitions [12]. A study of 29 participants performing shoulder abduction exercises demonstrated that, at follow-up after two weeks of unsupervised home-based exercises, less than $25 \%$ of the participants followed the instructions for time-under-tension (TUT) and performed correctly the exercise [10]. The main concern for patients not receiving the prescribed exercise dosage is that decisions on further progression or cessation of a specific program are difficult. The lack of objective measurements limits the ability of clinicians and researchers to evaluate the outcome of exercise interventions. This makes it virtually impossible to ascertain if lack of improvement is due to incorrect exercise, dosage, or poor adherence [12]. Therefore, an exercise monitoring system is necessary and useful for home-based trainees to quantify the exercise dosage, and most importantly, to improve adherence to the training program. sets and repetitions, rest periods, TUT, velocity, and muscle actions are important variables for monitoring [13], as they are of crucial importance for strength and power adaptation $[14,15,16]$. The number of repetitions and sets, as well as the TUT can be quantified using sensors, such as the BandCizer (BandCizer Aps, Denmark) [11] and a stretch sensor [17]. The BandCizer is a valid tool that can quantify contraction time, the number of repetitions performed, and the force used to stretch the elastic band $[11,18]$. However, the BandCizer uses two paired magnets to measure the deformation of the band and the deformation is susceptible to factors such as sensor placement [18]. Furthermore, since different muscle action phases have different training effect on muscles, differentiation between contraction-specific TUT may be important in some cases

PeerJ reviewing PDF | (2019:07:39592:3:1:NEW 30 Jan 2020) 
102 [18]. Dynamic muscular strength improvements were maximal when eccentric (ECC) action was

103

104

105

106

107

108

109

110

111

112

113

114

115

116

117

118

119

120

121

122

123

124

125

126

127

128

129

130

131

132

133

134

135

136 included in the training program $[14,19]$. Few studies have discussed how to segment sensor data based on muscle actions. Rathleff et al. developed a custom-written MATLAB program to segment stretch-sensor data into concentric (CON), quasi-isometric, and ECC phases and calculated the TUT of each contraction phase [10,20]. However, it should be noted that in their program, the assessor who rated the data needed to manually select the start and end points of each contraction phase, which led to program inefficiency. It required, on average, 55 seconds to determine total TUT for 10 repetitions from the stretch-sensor recordings and 3 10 minutes to rate the contraction-phase specific TUT for 10 repetitions [20].

In general, exercise is accompanied by muscle fatigue $[21,22]$. The fatigue and the recovery rate reflect the metabolic capacity of the patient, providing the physiotherapist with an intuitive perception of the subject's exercise capacity. Moreover, fatigue experienced by the subjects may decrease their compliance to exercise programs. Individuals who report high levels of fatigue are especially likely to drop out of an exercise program [22]. Numerous methods have been proposed to monitor muscular fatigue associated with exercise $[23,24]$ and surface electromyography (sEMG) is the most common approach used [23,25,26,27]. The regression slope of the linear regression of median frequency has been used as an important muscle fatigue index [26]. Recently, some fatigue-recovery protocols were deliberately designed to study the fatigability and the recovery ability of knee extensor muscle under intermittent, isometric, or dynamic maximal voluntary contractions in old adults $[28,29,30]$. Knee extension torque and power were measured using a dynamometer and the fatigability and recovery ability were expressed as reductions and increases in torque, velocity, and power.

Considering all these factors, the main objective of this study was to present a simple and automated method for calculating the repetition number and contraction phase-specific TUT during elbow flexor resistance training. Therefore, the biceps curl with a resistance band was selected, since it is a typical single-joint exercise focused on the strength and power improvement of elbow flexor muscles, and it is simple and suitable for home-based resistance training. A pilot experiment including 6 subjects was conducted to evaluate performance of the segmentation algorithm, and some fatigue and recovery indexes were proposed to quantify a subject's acute fatigue and recovery response to intermittent submaximal contraction (ISC).

Materials \& Methods

Concepts and big picture 
137

138

139

140

141

142

143

144

145

146

147

148

149

150

151

152

153

154

155

156

157

158

159

160

161

162

163

164

165

166

167

168

169

170

171

172

\section{2}

Accommodating resistance devices, such as bands and chains, are useful methods to maximize gains in strength and hypertrophy [14]. A significant feature of these devices is the varying resistance throughout the range of motion, where the level of resistance varies according to the rate and the maximum stretch of the material (greater stretch produces greater resistance), and similar results were obtained when resistance training was performed using traditional equipment (machines and weights) [31]. Accordingly, in this study the biceps curl with a resistance band was selected as the training exercise. Fig. 1 illustrates the concepts and flow chart of the system. Firstly, a framework for automatically segmenting force data into small segments based on muscle actions was built. Then, several indexes were introduced to quantify a subject's fatigue and recovery response during ISC.

As illustrated in Fig. 1, the subject sits on a wheelchair while performing the biceps curl exercise against resistance produced by a custom-made resistance band. A load cell is connected to the band to measure the resistance. Analog signals corresponding to the resistance were sampled and digitized by an analog-to-digital (A/D) converter and the data were sent to a desktop computer through an Arduino board. A graphical user interface (GUI) was built for monitoring the training process, and the force signals were acquired and saved for further analysis and post-processing. First, a peak detection method was used to find signal valleys, to divide the force data into small segments $(\mathrm{Fi}(\mathrm{t}))$, then the number of repetitions was calculated. In each segment, the first order derivative of force versus time was numerically calculated, and the maximum and minimum values were taken as the discriminating feature to segment the training cycle into the CON, ECC, and isometric (ISOM) contraction phases. The average force (Fave(i)) during ISOM contraction was calculated and the decreasing slopes ( $\mathrm{k} 1$ and $\mathrm{k} 2$ ) were defined as important muscle fatigue indexes. The recovery of normalized average force after 1minute rest (1MinRec) represented the fatigue recovery ability of the elbow flexor muscle.

\section{Measuring system}

Figure 2 shows the experiment set-up. A standard wheelchair is utilized to help the subject sit in a stable position and to help him/her lean the left or right arm against the armrest of the wheelchair. The subject flexes his/her forearm in the sagittal plane against the resistance produced by the custom-made resistance band. Fig. 3 illustrates details of the sensor, band, and Arduino board. In resistance training, the subject pulls the handle which is connected to the band. The band is attached to the load cell, which is anchored to the footplate of the wheelchair by a lifting hook. As mentioned above, a load cell (SC301A, $100 \mathrm{~kg}$ ) was used to measure the time- 
173 varying resistance. Analog signals were converted to digital signals through an A/D converter

174 (AE-HX711-SIP, $30 \mathrm{~Hz}$ ). The load cell was calibrated and the scale factor was calculated before

175 the experiments using calibration weights. A personal computer (PC) was used to acquire,

176 display, save, and process the force data through a serial COM port. Since the training load was

177 different among different trainees, three different types of band (Green, Red, and Yellow) were

178 constructed and connected to the handle using a carabiner clip. As shown in Fig. 4, each band

179 was tested under uniaxial tensile extension, and the force data were recorded to obtain the load

180 versus extension curve. Based on the curves, it was observed that the green band had the highest

181 resistance, while the yellow band had the lowest. In our study, different combinations of bands

182 were used to set different training loads for the trainees according to their muscle strength.

183

184

185

186

187

188

189

190

191

192

193

194

195

196

197

198

199

200

201

202

203

204
*INSERT FIGURE 2 AROUND HERE*

*INSERT FIGURE 3 AROUND HERE*

*INSERT FIGURE 4 AROUND HERE*

\section{Force data segmentation}

The proposed algorithm incorporates repetition segmentation and muscle action segmentation. The repetition segmentation is a pre-processing stage, where the force data are divided into individual segments according to the training cycle. Fig. 5 illustrates a typical plot of resistance data during the biceps curl exercise. As shown by the curve, the force signal exhibits strong periodicity with distinct peaks and troughs. A simple and efficient peak detection method [32] was used to find the minimum value of the trough, which was taken as the segmentation point. In the segmentation process, the mean resistance value was used as the threshold value to divide the force signal into peaks and troughs. An example of segmentation results is illustrated in Fig. 6.

*INSERT FIGURE 5 AROUND HERE*

*INSERT FIGURE 6 AROUND HERE* 
205

206

207

208

209

210

211

212

213

214

215

216

217

218

219

220

221

222

223

224

225

226

227

228

229

230

231

232

233

234

235

236

237

238

239

240

241

Subsequently, a muscle action segmentation algorithm was proposed to divide each biceps curl cycle into four time-windows. The biceps curl exercise is an easy-to-recognize movement, which incorporates all the CON, ISOM, and ECC muscle actions. However, in general there is no clear cut-off point between CON, ISOM, ECC, and rest phases, since training is a complex process that is affected by many subjective factors, such as personal ability, willingness, and fatigue. Fig. 7 presents a visual representation of how the segmentation algorithm operates. The core idea of the segmentation algorithm is based on the difference in force derivatives at the different contraction phases. Raw force data containing one flexion-extension repetition were obtained using the repetition segmentation algorithm proposed in the previous paragraph. Firstly, the first order derivative of force versus time was numerically calculated. From the derivative curve, it was perceived that the maximum and minimum values were the most discriminating features. During the CON contraction, the force-derivative increases rapidly at the beginning, reaches a maximum value, and then rapidly decreases to zero. In ISOM contraction, the subject keeps his/her forearm in quasi-static state for a few seconds and the force-derivative does not change much. During the ECC contraction, the subject releases his/her forearm and the forcederivative reaches a minimum value. Consequently, the maximum and minimum values of the force-derivative were considered as important thresholds to segment the force data. The segmentation model can be expressed as follow:

$F(t) \in\left\{\begin{array}{l}C O N, \quad\left(0.1^{*} \operatorname{Max} \leq d F(t) \leq \max \right) \\ I S O M,\left(0.1^{*} \operatorname{Min}<d F(t)<0.1^{*} \text { Max }\right) \\ E C C, \quad(\operatorname{Min} \leq d F(t) \leq 0.1 * \text { Min }\end{array}\right.$

\section{*INSERT FIGURE 7 AROUND HERE*}

During the CON and ECC contraction, data with a derivative greater or less than $10 \%$ of the maximum and minimum values were chosen and the trend line was calculated to approximate the change in force during these contraction phases. The trend line is an approximate alternative of the data in the contraction phases. On the one hand, this approximation eliminates some accidental situations in the data, such as fluctuations or slacks. On the other hand, when the forearm flexes or extends, the force tendency is preserved as much as possible. The trend line (y $=\mathrm{fi}(\mathrm{t}), \mathrm{i}=1 \sim 3)$ was calculated using linear regression. The trend line intersections $(\mathrm{A} \sim \mathrm{D})$ and the line of constant function $(\mathrm{y}=0)$ divided the arm biceps curl exercise into the CON, ECC, and ISOM contraction phases and the rest period.

\section{Extraction of Fatigue and Recovery Indexes}

In general, exercise is accompanied by muscle fatigue. When fatigue occurs, the threshold to trigger action potentials in a motor unit increases, i.e., the motor unit's tendency to fire decreases [33,34]. Since the discharge rate changes, the number of recruited motor units is reduced, resulting in the reduction of muscle force. Here, muscle fatigue was defined as the 
242 relative decline in average force (Fave(i)) during ISOM contraction and the decreasing slopes

243 ( $\mathrm{k} 1$ and $\mathrm{k} 2$ ) of Fave(i) were used as index of fatigability. The recovery of Fave(i) after 1-minute 244 rest (1MinRec) was used to quantify subject's ability to recover from fatigue.

245

246

247

248

249

250

251

252

253

254

255

256

257

258

259

260

261

262

263

264

265

266

267

268

269

270

271

272

273

274

275

276

277

278

279

Figure 8 presents how the decreasing slopes and the recovery of the average force after rest were calculated. The average force during ISOM contraction is expressed as follow:

$F_{\text {ave }}(i)=\frac{B_{i}(y)+C_{i}(y)}{2}$

where $\mathrm{Bi}(\mathrm{y})$ and $\mathrm{Ci}(\mathrm{y})$ represent the $\mathrm{y}$ value of the starting and ending point of ISOM, respectively. The regression slopes $\mathrm{k} 1$ and $\mathrm{k} 2$ were calculated using a regression function. The calculation of 1 MinRec is expressed as follow:

1 MinRec $=\frac{P 2(y)-P 1(y)}{F 0} \times 100 \%$

where $\mathrm{P} 2(\mathrm{y})$ and $\mathrm{P} 1(\mathrm{y})$ represent the $\mathrm{y}$ value of points $\mathrm{P} 1$ and $\mathrm{P} 2$, respectively, and F0 represents the initial force.

\section{*INSERT FIGURE 8 AROUND HERE*}

\section{Experimental Procedures}

Figure 9 gives a visual representation of the procedure followed in the fatigue and recovery protocol. The protocol has been carefully designed to incorporate four parts: one-repetition maximum (1RM) test, 5-minutes ISC, 1-minute rest, and 2-minutes ISC. The 1RM test is performed to assess subject's flexor muscle strength and the obtained value is the basis for selecting the combination of elastic bands. During the 1RM test, the number of bands was empirically increased to reach the maximum load the subject can pull while flexing his/her forearm throughout the full range of motion (elbow joint angle more than $150^{\circ}$ ). Three sets of contractions were performed, and the average force was calculated as the final 1RM. During the 5-minutes ISC, the number of elastic bands was chosen to ensure that the initial average force was $40-60 \%$ that of $1 \mathrm{RM}$. ISC is an intermittent and dynamic voluntary contraction process with many repetitions. During each repetition, the subject flexes his/her forearm to a maximum angle (CON contraction), maintains the angle for about 3 seconds (ISOM contraction), and then returns to a relaxed state (ECC contraction). A short rest was allowed between each repetition and the rest time was usually no more than 4 seconds. During the 1-minute rest, the subject was encouraged to stand up from the wheelchair and swing his/her arm to recover from the fatigued state. The 1-minute rest and 2-minutes ISC were performed in succession after the 5-minutes ISC. The subjects performed the exercises with both hands and were given ample rest between experiments. All subjects were right-handed. A training session was conducted in order to get the subjects familiar with the experimental procedure before the formal experiment. In addition, the subjects performed the biceps curl exercise against resistance band during the 5-minutes and 2minutes ISC. The biceps curl movement consisted of the motion of elbow joint flexing and 
280

281

282

283

284

285

286

287

288

289

290

291

292

293

294

295

296

297

298

299

300

301

302

303

304

305

306

307

308

309

310

311

312

313

314

315

316

317

318

extending while the wrist joint was fixed in a neutral position (no flexion or extension). Flexion represented the movement of elbow flexion and extension represented the movement of elbow extension.

At this stage, we aimed to see if the actual monitoring system was able to detect the contraction phases, and the ability of the subjects to use the prescribed TUT in the ISOM phase (3 seconds). Therefore, in this study, the experiment instructor was required to accompany the subject to complete the entire experiment. During the experiment performed by each subject, the instructor was required to start the measurement system, click the "Record" button and remind the subject about the start and end time of the entire experiment, as well as the 1-minute rest period. Subjects were told to keep the TUT in the ISOM phase for 3 seconds and a metronome was used to guide on the TUT in ISOM phase. The metronome provided guidance for the subject, not the instructor.

\section{*INSERT FIGURE 9 AROUND HERE*}

\section{Statistical Analyses}

Statistical analysis was carried out in Excel (Microsoft Office 2010, Microsoft) and with the aid of MATLAB (R2012b). Descriptive statistics were performed to subjects' demographic data. The AVERAGE and STDEV.S functions were used to calculate the mean and standard deviation of subjects' personal information, respectively. The contraction-phase specific TUT was calculated using MATLAB. Student's test was performed to compare the difference in decreasing slope and $1 \mathrm{MinRec}$ between left and right hand. Significance test for the alpha value was set at 0.05 .

\section{Participants}

Some formalities were required to obtain an ethical license to allow experiments with the elderly population. Moreover, unlike healthy individuals, the equipment used by older individuals needs to be safer and more reliable. Therefore, even the case of this paper was particularly made for an older demographic, healthy subjects were recruited to show the performance of our segmentation algorithm. Six healthy subjects ( 3 males and 3 females, age $27.3 \pm 4.2$ years, weight $60.3 \pm 11.7 \mathrm{~kg}$, height $171 \pm 9.7 \mathrm{~cm}$ ) volunteered to participate in this pilot experiment. Participant recruitment and experiments were conducted in the affiliate of the first author, Micro Mechatronics Laboratory, Faculty of Civil Aviation and Aeroautics, Kunming University of Science and Technology, Kunming, China. All subjects were familiarized with the research procedures and gave their informed consent. None of the subjects reported known neurological, musculoskeletal, or orthopedic disorders. Demographic data of the study participants are presented in Table 1. 
320

321

322

323

324

325

326

327

328

329

330

331

332

333

334

335

336

337

338

339

340

341

342

343

344

345

346

347

348

349

350

351

\section{Human Ethics}

The following information was supplied relating to ethical approvals (i.e., approving body and any reference numbers):

This study was approved by the Medical Ethics Committee of the Kunming University of Science and Technology, Kunming, China (approval number: 2019YXY06). Signed informed consent was obtained from all study participants prior to data collection.

\section{Results}

Figure 10 shows a typical force-time curve of a male subject. As dynamic contraction progressed, the maximum force the subject could reach reduced gradually and was restored to a certain extent after the 1-minute break. When participants became exhausted, there were some fluctuations in force during ISOM contraction. Other details about the experiment, including the initial force, are presented in Table 2. As described in the previous section, the combination of resistance bands was chosen based on a subject's muscle strength. Furthermore, the repetition numbers before rest (m) and after rest (n) were calculated and are presented in Table 2.

\section{*INSERT FIGURE 10 AROUND HERE*}

*INSERT TABLE 2 AROUND HERE*

\section{Contraction-phase specific TUT}

The proposed segmentation algorithm was successfully applied with the aid of MATLAB and, after all participants had completed the experiment, TUT was calculated. The calculated contraction-phase specific TUT could be applied to investigate whether participants followed the prescribed home-based program, and to evaluate performance of the segmentation algorithm. Table 3 reports the proportion of contraction-phase specific TUT and the total TUT of all subjects. The total TUT is the sum of TUT during CON, ISOM, and ECC contractions. The proportion of TUT varied greatly between subjects. The subjects received very different contraction-phase specific and total TUT, even though the total training time was the same. Differences in TUT make it difficult for clinicians and researchers to interpret the training results and state if the subject receives the prescribed training volume [10]. In addition, even if a metronome was used to provide guidance on the TUT, the average TUT (3.75 $\pm 0.62 \mathrm{~s})$ during 
352 ISOM was longer than 3 seconds, meaning that most of the subjects did not perform the exercise 353 as prescribed [10]. The differences in TUT emphasize the importance and necessity of

354 monitoring the exercise dose during home-based resistance training.

355

356

357

358

359

360

361

362

363

364

365

366

367

368

369

370

371

372

373

374

375

376

377

379 380

381

383
378

382

*INSERT TABLE 3 AROUND HERE*

\section{Elbow flexor fatigue and recovery profiles}

The acute fatigue response could be investigated to explain how fatigue experience affected the compliance of patients as it is believed that fatigue experience may decrease a patients' motivation to participate in a training program [21,22]. Here, we present the calculated elbow flexor fatigue and recovery profiles during the training to prove the capability of the system in monitoring the acute fatigue response. The average force during ISOM contraction was calculated using the aforementioned algorithm. The force data distributions and the resulted trend lines for all participants are shown in Fig. 11. For the purpose of comparison, all force data were normalized to the initial value $[29,30,35,36]$. The decreasing slopes of the average force before and after rest in female and male subjects were analyzed and the statistical result is given in Table 4. Table 5 reports the statistics of average force recovery in left and right hand after 1minute rest.

It shown in Fig. 11, and stated in many references [29,37], all definitions of fatigue necessitate a decline in force or power, the average force during ISOM contraction decreased continuously. The Student's t test revealed that no significant differences were observed in the decreasing slope between left and right hands $(p=0.2$ and $p=0.14$, respectively, Table 4$)$. The average force restored to a certain extent after the 1-minute rest, however it decreased much faster than before the 1-minute rest. This was probably because the 1-minute rest does not prevent the development of central fatigue [38].

*INSERT FIGURE 11 AROUND HERE* *INSERT TABLE 4 AROUND HERE* 
385

386

387

388

389

390

391

392

393

394

395

396

397

398

399

400

401

402

403

404

405

406

407

408

409

410

411

412

413

414

415

416

417

418

\section{Discussion}

In the previous sections, the measurement system was described and a segmentation algorithm that automatically segments the biceps curl cycle into small parts based on muscle actions was proposed. A pilot experiment with 6 subjects was conducted and the repetition number and contraction-phase specific TUT were calculated and compared. Some fatigue and recovery indexes were proposed in order to show fatigue-recovery profiles of elbow flexor for all subjects. Furthermore, in the following sections, the performance of the segmentation algorithm was evaluated and the application prospect of the instrument in a practical setting was discussed.

\section{Performance of the Segmentation Algorithm}

To the best of our knowledge, this is the first study that uses an automated method to rate the contraction-phase specific TUT. Compared to other manual methods [10,20], this method exhibited high efficiency. Using a dual-core $3.5 \mathrm{GHz}$ Intel processor laptop, the rating time for one trial takes no more than 5 seconds. Some typical segmentation results are presented in Fig. 12. As demonstrated, the proposed algorithm can correctly segment the force data into the four contraction phases (CON, ISOM, ECC, and Rest), even when there are some fluctuations or slacks between two contraction phases. In some cases, when the trainee bent his/her arm at the beginning of CON, some failed startups occurred. During those failed startups, the resistance was relatively small and these data did not produce effective TUT. In such cases, the algorithm removed these invalid data and used the trend line to determine the most likely starting point. In other cases, the boundary between two contraction phases was clearly marked by the trend line intersection. The segmentation results were visually checked and it was found that the algorithm successfully segmented all the data.

\section{*INSERT FIGURE 12 AROUND HERE*}

To further verify the correctness of the algorithm, two assessors, who were unfamiliar with the experiment, were recruited to manually pick the visually observed cut-off point between two contraction phases, and the calculation results of TUT from the two assessors were used as gold standard for assessing the TUT results from our segmentation algorithm. The TUT comparison is demonstrated in Fig. 13, and shows good TUT agreement and contraction segment agreement between the proposed algorithm and the assessor. The total TUT was exactly the same, however the algorithm's TUT was a little longer than that of the assessors during CON and ECC, while during ISOM, the algorithm's TUT was a little shorter than that of the assessors. In addition, the 
419

420

421

422

423

424

425

426

427

428

429

430

431

432

433

434

435

436

437

438

439

440

441

442

443

444

445

446

447

448

449

450

451

452

comparison results showed that there were differences in the rating results between assessors, thereby indicating that the rating of the data was subjectively influenced by the assessor. The proposed segmentation algorithm was implemented programmatically, avoiding the subjectivity of the assessors. Therefore, it is an automated and objective method for quantifying the exercise dosage during home-based elbow flexor resistance training.

\section{*INSERT FIGURE 13 AROUND HERE*}

\section{Application prospect of the instrument in a practical setting}

The hypothetical application scenario of this instrument is to help people who were confined to a wheelchair or bed to perform elbow flexor resistance training. Compared with healthy subjects, those people have deficits in their physiological system, and are more sensitive to the training load and volume. Consequently, a custom-made resistance band shown in Fig. 3 was designed and made to monitor the training dose and acute fatigue responses. In this study, we only showed the example of using biceps curl exercise with resistance bands to regain elbow flexor muscle strength in people who were confined to a wheelchair or bed. However, this system can be applied easily to other more functional movements, including forearm extension or elbow extensor resistance training exercise. When a trainee holds the resistance band and performs training exercise, an emerging problem is how to fix the other end. By changing the way how the other end is fixed, this method can be applied to many other movements. Moreover, as presented in Fig. 3, the measuring system is easy to use and cheap, indicating its broad application prospects. Presently, only two resistance bands were made, one is applied for testing at an elderly rehabilitation center. Additional bands will be prepared with the assistance of sporting goods companies to meet the safety and reliability requirements of rehabilitation centers for elderly people. In addition, the segmentation algorithm was implemented in MATLAB. A website version of the segmentation algorithm will help more people to freely and conveniently use this algorithm. Moreover, we are currently designing a novel measuring system, which allows for automatically uploading the resistance force to the website through a single-board computer. Through the novel measuring system and website interface, therapists will be able to remotely monitor the training dose of the home-base trainee and more conveniently obtain experimental data. In conclusion, additional studies will be needed before our approach can be applied to a wider range of practical applications.

\section{Limitations and future work}


453

454

455

456

457

458

459

460

461

462

463

464

465

466

467

468

469

470

471

472

473

474

475

476

477

478

479

480

481

482

483

484

485

A limitation of this pilot study was its small sample size in the experiment. Only 6 healthy subjects volunteered to participate in the experiment, and the small sample size may have weakened the application of some conclusions to a larger population. With additional time and funding, future studies with larger sample size, including participants from a wider population, will address this limitation. In addition, the final goal of this project was to establish an automatic monitoring system for home-based resistance training. At this stage, we aimed to see if the actual monitoring system was able to detect the contraction phases and detect the ability of the subjects to use the prescribed TUT in the ISOM phase ( 3 seconds). We did not investigate how the fatigue affects compliance of patients. However, since the fatigue experience will affect patients' compliance to training programs, how and to what extend the fatigue experience affect patients' compliance will be investigated in our future work.

\section{Conclusions}

In summary, this study presented an automated method for segmenting resistance data into contraction phase-specific segments during elbow flexor resistance training, for establishing an automatic monitoring system for home-based resistance training. The principle of the algorithm was described in detail, and experiments were performed to evaluate the performance of the method. The good agreement in TUT measurements between the proposed algorithm and the assessors indicated that the proposed algorithm can correctly segment the contraction into contraction phase-specific parts, thus providing clinicians and researchers with an automated and objective method for quantifying home-based elbow flexor resistance training. The objective and automated nature of the segmentation algorithm has the advantage of eliminating the subjective influence of the assessor and improving the rating efficiency. In addition, some fatigue and recovery indexes were proposed in order to show fatigue-recovery profiles of elbow flexor.

\section{Acknowledgements}

The authors are very grateful to Msrh Izza and Lurui Wang for rating the experimental data.

\section{References}

[1] Liu, Chiung-ju, and Nancy K. Latham. "Progressive resistance strength training for improving physical function in older adults." Cochrane database of systematic reviews 3 (2009).

[2] Michael L. Pollock, Glenn Gaesser, Janus D. Butcher, Jean Pierre Després, Rod K. Dishman, Barry A. Franklin, Carol Ewing Garber. "The recommended quantity and quality of exercise for 
486 developing and maintaining cardiorespiratory and muscular fitness, and flexibility in healthy

487 adults." Medicine and science in sports and exercise 30.6 (1998): 975-991.

488 [3] American College of Sports Medicine. "American College of Sports Medicine position stand. 489 Progression models in resistance training for healthy adults." Medicine and science in sports and 490 exercise 41.3 (2009): $687 . \mathrm{h}$

491 [4] Schutzer, Karen A., and B. Sue Graves. "Barriers and motivations to exercise in older adults." 492 Preventive medicine 39.5 (2004): 1056-1061.

493 [5] Thiebaud, Robert S., Merrill D. Funk, and Takashi Abe. "Home-based resistance training for 494 older adults: a systematic review." Geriatrics \& gerontology international 14.4 (2014): 750-757.

495 [6] Iman Khaghani Far, Michela Ferron, Francisco Ibarra, Marcos Baez, Stefano Tranquillini, 496 Fabio Casati, Nicola Doppio. "The interplay of physical and social wellbeing in older adults:

497 investigating the relationship between physical training and social interactions with virtual social 498 environments." PeerJ Computer Science 1 (2015): e30.

499 [7] Wilson, Richard D., Steven A. Lewis, and Patrick K. Murray. "Trends in the rehabilitation 500 therapist workforce in underserved areas: 1980-2000." The Journal of Rural Health 25.1 (2009): $50126-32$.

502 [8] Dobkin, Bruce H. "A rehabilitation-internet-of-things in the home to augment motor skills 503 and exercise training." Neurorehabilitation and neural repair 31.3 (2017): 217-227.

504 [9] M S Rathleff, E M Roos, J L Olesen, S Rasmussen. "Exercise during school hours when 505 added to patient education improves outcome for 2 years in adolescent patellofemoral pain: a 506 cluster randomised trial." Br J Sports Med 49.6 (2015): 406-412.

507 [10] Mathilde Faber, Malene H. Andersen, Claus Sevel, Kristian Thorborg, Thomas Bandholm, 508 Michael Rathleff. "The majority are not performing home-exercises correctly two weeks after 509 their initial instruction — an assessor-blinded study." PeerJ 3 (2015): e1102.

510 [11] Riel H, Matthews M, Vicenzino B, Bandholm T, Thorborg K, Rathleff MS. "Feedback 511 Leads to Better Exercise Quality in Adolescents with Patellofemoral Pain." Medicine and science 512 in sports and exercise 50.1 (2018): 28-35.

513 [12] Michael S Rathleff, Thomas Bandholm, Kate A McGirr, Stine I Harring, Anders S 514 Sørensen, Kristian Thorborg. "New exercise-integrated technology can monitor the dosage and 515 quality of exercise performed against an elastic resistance band by adolescents with 516 patellofemoral pain: an observational study." Journal of physiotherapy 62.3 (2016): 159-163. 
517 [13] Brendan R. Scott, Grant M. Duthie, Heidi R. Thornton, Ben J. Dascombe. "Training 518 monitoring for resistance exercise: theory and applications." Sports Medicine 46.5 (2016): 687519698.

520 [14] Kraemer, William J., and Nicholas A. Ratamess. "Fundamentals of resistance training: 521 progression and exercise prescription." Medicine \& Science in Sports \& Exercise 36.4 (2004): $522674-688$.

523 [15] Bird, Stephen P., Kyle M. Tarpenning, and Frank E. Marino. "Designing resistance training 524 programmes to enhance muscular fitness." Sports medicine 35.10 (2005): 841-851.

525 [16] Crewther, Blair, John Cronin, and Justin Keogh. "Possible stimuli for strength and power 526 adaptation: acute metabolic responses." Sports Medicine 36.1 (2006): 65-79.

527 [17] Simon L. Kappel, Michael S. Rathleff, Dan Hermann, Ole Simonsen, Henrik Karstoft and 528 Peter Ahrendt. "A novel method for measuring in-shoe navicular drop during gait." Sensors 12.9 529 (2012): 11697-11711.

530 [18] Rathleff Michael S., Thorborg Kristian, Rode Line A., McGirr Kate A., Sørensen Anders S., 531 Bøgild Anders, Bandholm Thomas. "Adherence to commonly prescribed, home-based strength 532 training exercises for the lower extremity can be objectively monitored using the Bandcizer." 533 The Journal of Strength \& Conditioning Research 29.3 (2015): 627-636.

534 [19] Komi, P. V., M. Kaneko, and O. Aura. "EMG activity of the leg extensor muscles with 535 special reference to mechanical efficiency in concentric and eccentric exercise." International 536 Journal of Sports Medicine 8.S 1 (1987): S22-S29.

537 [20] Rathleff, Michael Skovdal, Kristian Thorborg, and Thomas Bandholm. "Concentric and 538 eccentric time-under-tension during strengthening exercises: validity and reliability of stretch539 sensor recordings from an elastic exercise-band." PloS one 8.6 (2013): e68172.

540 [21] Andrew, Geoege M., and John O. Parker. "Factors related to dropout of post myocardial 541 infarction patients from exercise programs." Medicine and Science in Sports 11.4 (1979): 376542378.

543 [22] John R. Hughes, Richard S. Crow, David R. Jacobs Jr., Maurice B. Mittelmark \& Arthur S. 544 Leon. "Physical activity, smoking, and exercise-induced fatigue." Journal of behavioral 545 medicine 7.2 (1984): 217-230.

546 [23] Haiwei Dong, Izaskun Ugalde, Nadia Figueroa and Abdulmotaleb El Saddik. "Towards 547 whole body fatigue assessment of human movement: A fatigue-tracking system based on 548 combined semg and accelerometer signals." Sensors 14.2 (2014): 2052-2070. 
549 [24] Al-Mulla, Mohamed R., Francisco Sepulveda, and Martin Colley. "A review of non550 invasive techniques to detect and predict localised muscle fatigue." Sensors 11.4 (2011): 35455513594.

552 [25] Nurhazimah Nazmi, Mohd Azizi Abdul Rahman, Shin-Ichiroh Yamamoto, Siti Anom 553 Ahmad, Hairi Zamzuri and Saiful Amri Mazlan. "A review of classification techniques of EMG 554 signals during isotonic and isometric contractions." Sensors 16.8 (2016): 1304.

555 [26] Chang, Kang-Ming, Shin-Hong Liu, and Xuan-Han Wu. "A wireless sEMG recording 556 system and its application to muscle fatigue detection." Sensors 12.1 (2012): 489-499.

557 [27] Al-Mulla, Mohamed R., and Francisco Sepulveda. "Novel feature modelling the prediction 558 and detection of semg muscle fatigue towards an automated wearable system." Sensors 10.5 559 (2010): 4838-4854.

560 [28] Callahan, Damien M., Stephen A. Foulis, and Jane A. Kent-Braun. "Age-related fatigue 561 resistance in the knee extensor muscles is specific to contraction mode." Muscle \& Nerve: 562 Official Journal of the American Association of Electrodiagnostic Medicine 39.5 (2009): 692563702.

564 [29] Callahan, Damien M., Brian R. Umberger, and Jane A. Kent. "Mechanisms of in vivo 565 muscle fatigue in humans: investigating age-related fatigue resistance with a computational 566 model." The Journal of physiology 594.12 (2016): 3407-3421.

567 [30] Christopher W. Sundberg, Andrew Kuplic, Hamidollah Hassanlouei, and Sandra K. Hunter.

568 "Mechanisms for the age-related increase in fatigability of the knee extensors in old and very old 569 adults." Journal of Applied Physiology (2018).

570 [31] Daniel Souzaa, Matheus Barbalhoa, Carlos Alexandre Vieiraa, Wagner Rodrigues Martinsb, 571 Eduardo L. Cadorec, Paulo Gentila. Minimal dose resistance training with elastic tubes promotes 572 functional and cardiovascular benefits to older women[J]. Experimental gerontology, 2019, 115: $573 \quad 132-138$.

574 [32] Kun-Hui Chen, Po-Chao Chen, Kai-Chun Liu and Chia-Tai Chan. "Wearable sensor-based 575 rehabilitation exercise assessment for knee osteoarthritis." Sensors 15.2 (2015): 4193-4211.

576 [33] Liu, Jing Z., Robert W. Brown, and Guang H. Yue. "A dynamical model of muscle 577 activation, fatigue, and recovery." Biophysical journal 82.5 (2002): 2344-2359.

578 [34] Enoka, Roger M., and Andrew J. Fuglevand. "Motor unit physiology: some unresolved 579 issues." Muscle \& nerve 24.1 (2001): 4-17. 
580 [35] Brennan J. Thompson, Eric D. Ryan, Trent J. Herda, Pablo B. Costa, Ashley A. Herda, Joel 581 T. Cramer. "Age-related changes in the rate of muscle activation and rapid force characteristics." 582 Age 36.2 (2014): 839-849.

583 [36] Ian R. Lanza, Danielle M. Wigmore, Douglas E. Befroy, Jane A. Kent-Braun. "In vivo ATP 584 production during free-flow and ischaemic muscle contractions in humans." The Journal of 585 physiology 577.1 (2006): 353-367.

586 [37] Sanchez-Medina, Luis, and Juan José González-Badillo. "Velocity loss as an indicator of 587 neuromuscular fatigue during resistance training." Medicine and science in sports and exercise $588 \quad 43.9$ (2011): 1725-1734.

589 [38] Bilodeau, Martin. "Central fatigue in continuous and intermittent contractions of triceps 590 brachii." Muscle \& Nerve: Official Journal of the American Association of Electrodiagnostic 591 Medicine 34.2 (2006): 205-213. 
Figure 1

Concepts and big picture of the system.

$\mathrm{Fi}(\mathrm{t})$ is the resistance in repetition $\mathrm{i} . \operatorname{Max}(\mathrm{i})$ and $\operatorname{Min}(\mathrm{i})$ are the maximum and minimum force slope values. CON, ECC, and ISOM are concentric, eccentric, and isometric contraction, respectively. Rest is the force data in the rest phase, Fave(i) is the average force during ISOM, used to represent the fatigue and recovery profiles of elbow flexor during dynamic contraction, and k1 and k2 are the decreasing slopes of Fave(i) before and after rest. 1 MinRec is the recovery of Fave(i) after 1-minute rest. Drawing source credit: Taojin Xu.

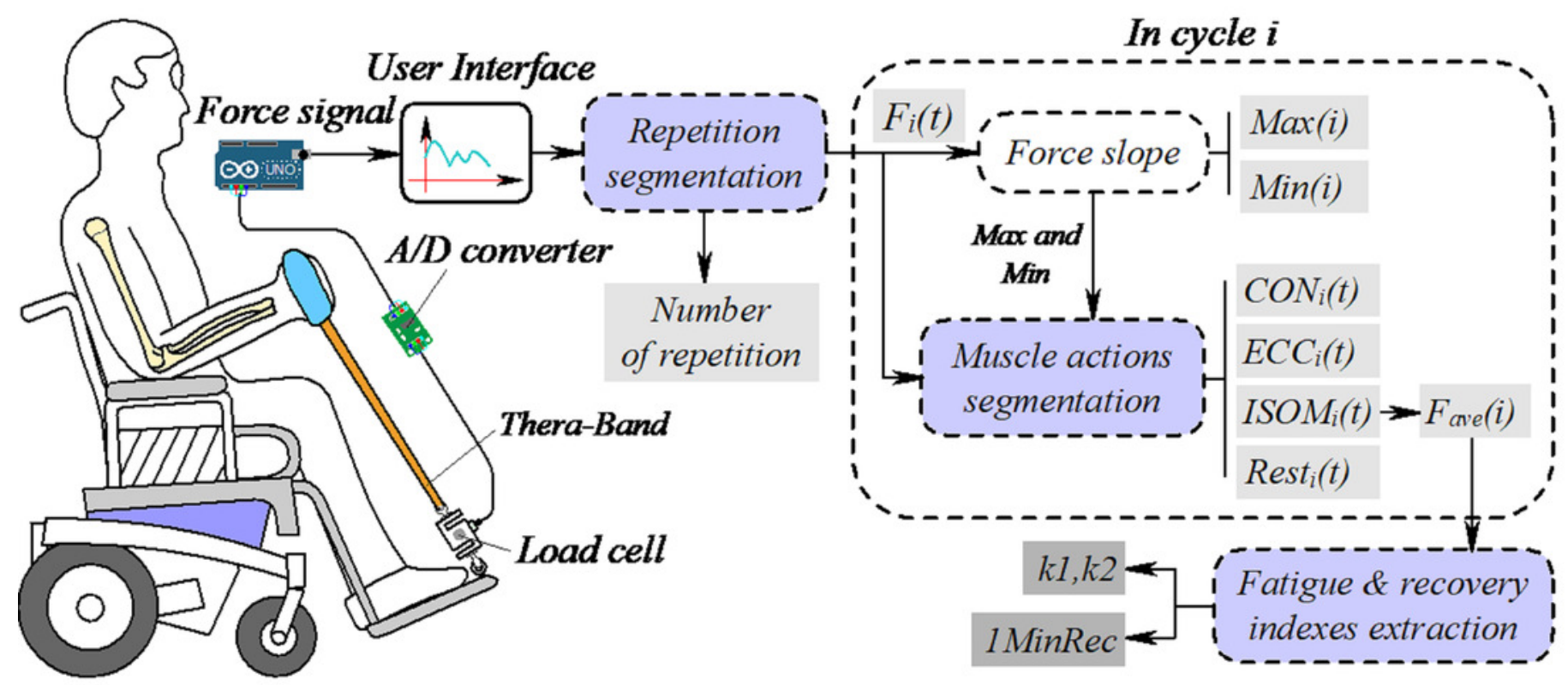


Figure 2

The experiment set-up.

Photo source credit: Taojin Xu.

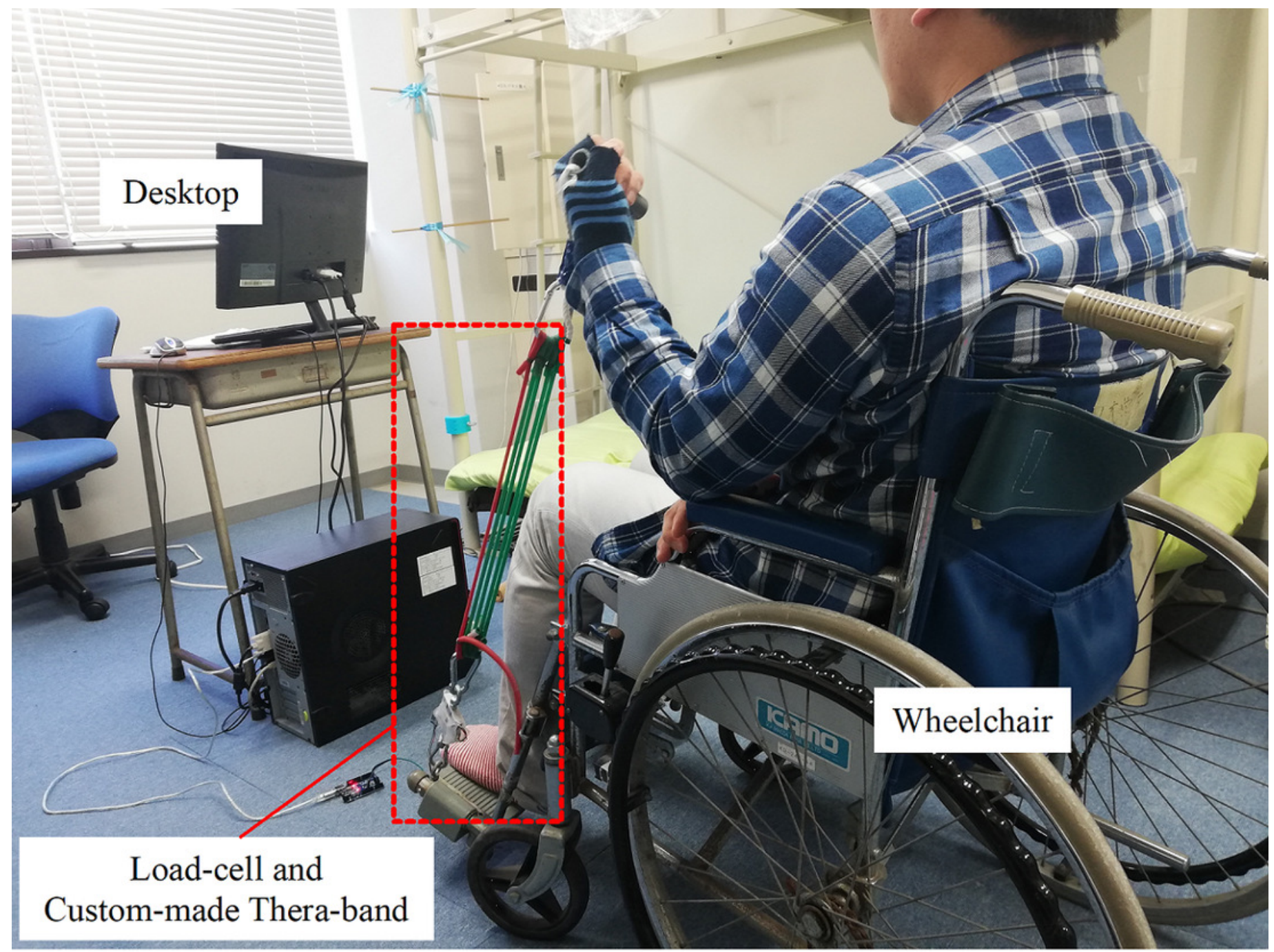


Figure 3

Details of the sensor, custom-made Thera-Band, and Arduino board.

Photo source credit: Taojin Xu.

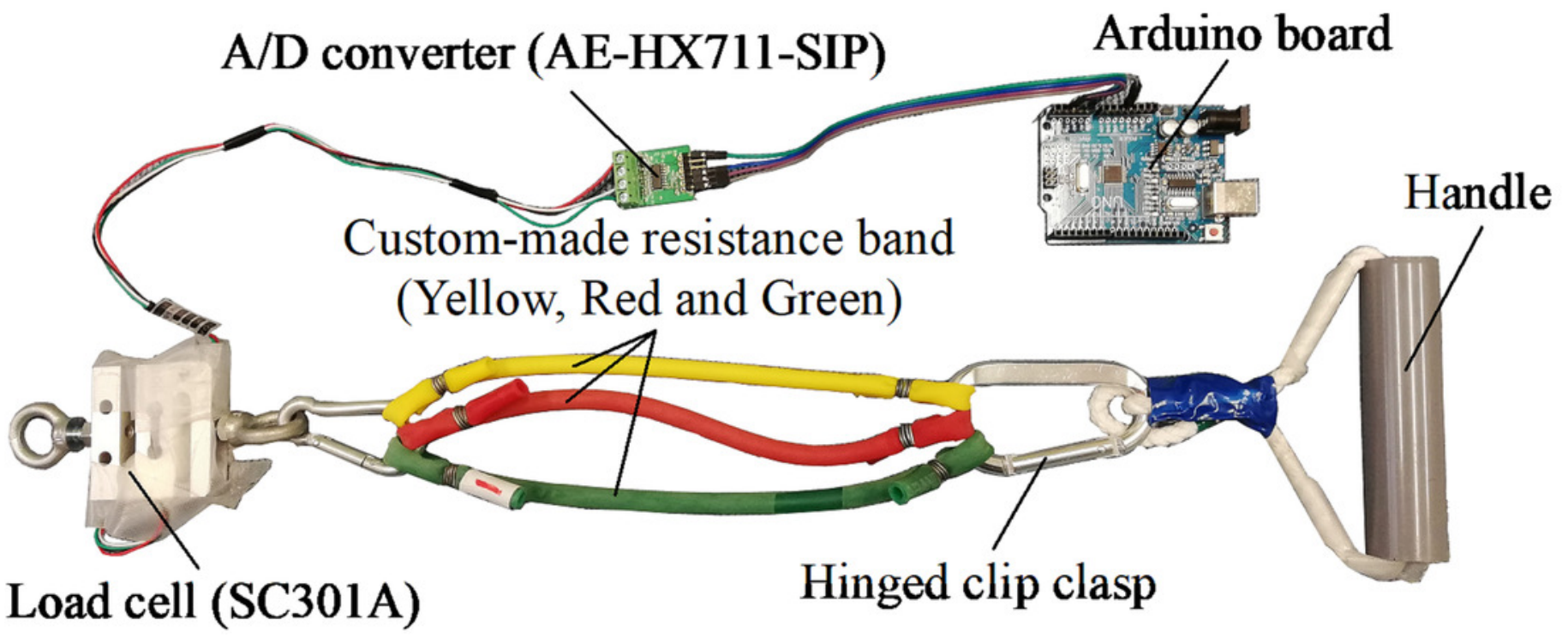


Figure 4

Load versus extension curves of the different custom-made Thera-Bands.

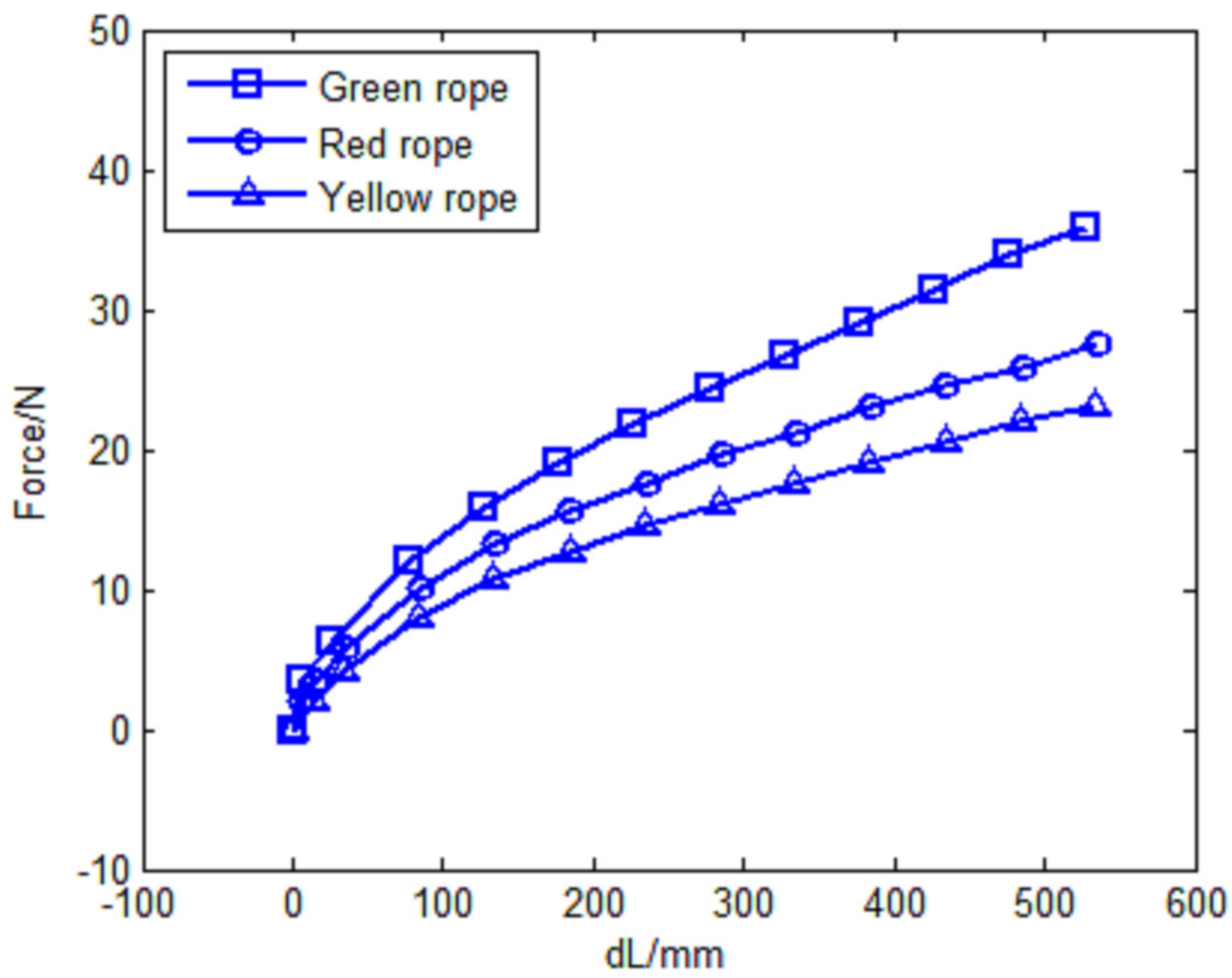


Figure 5

Segmentation points locating logic.

The minimum value of the trough was chosen as the segmentation point.

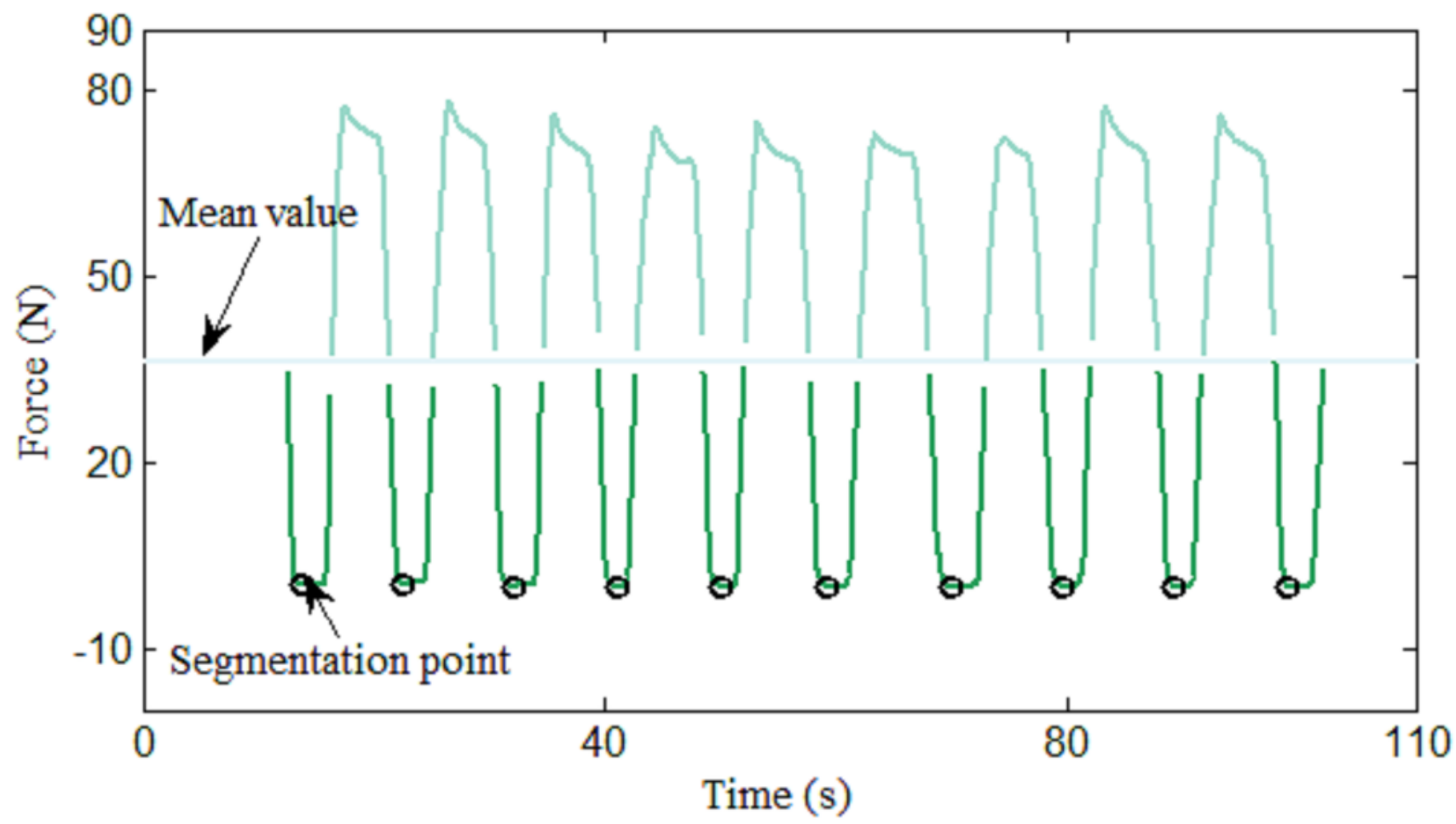


Figure 6

Example of a segmentation result.

Different training cycles are illustrated in different colors.

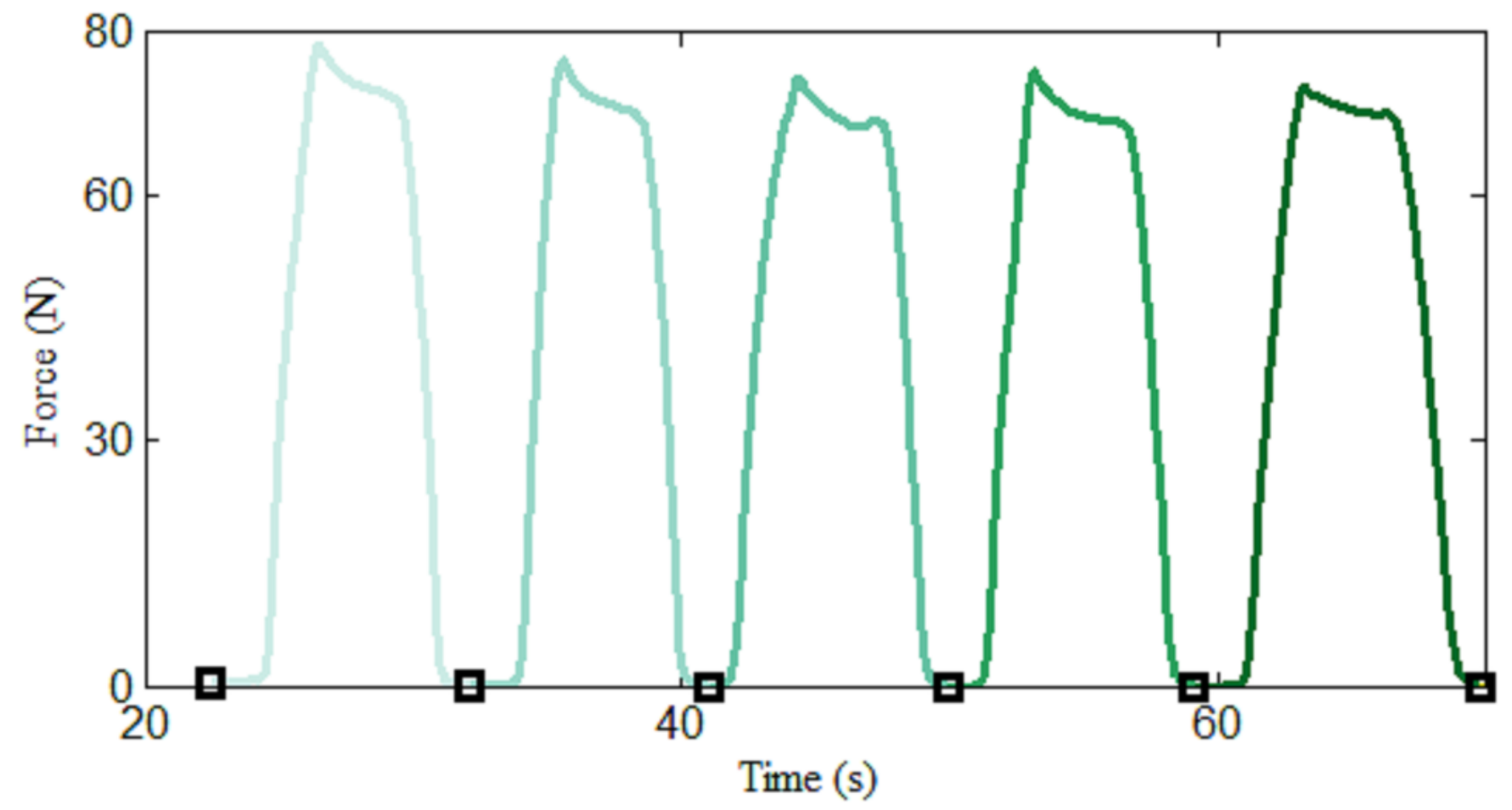


Figure 7

Schematic diagram of the segmentation algorithm.

(a) The maximum and minimum values of the first order derivative of force versus time were used as thresholds to divide the data into three parts. (b) Trend line intersections were used to segment the arm biceps curl exercise into four contraction phases (CON, ISOM, ECC and rest).

(a)

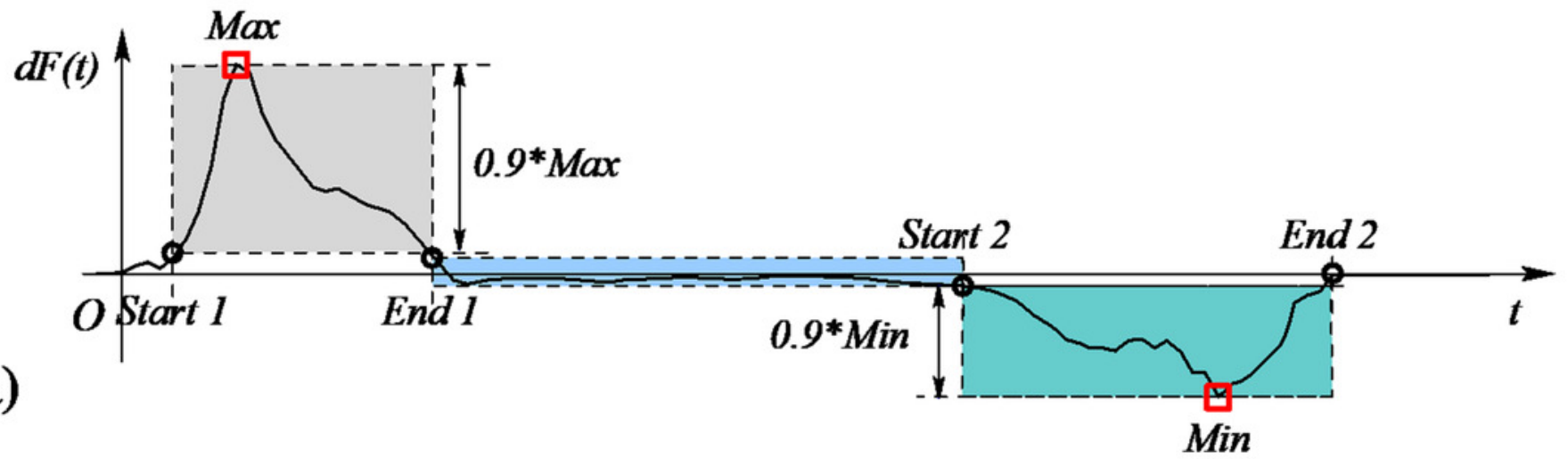

(b)

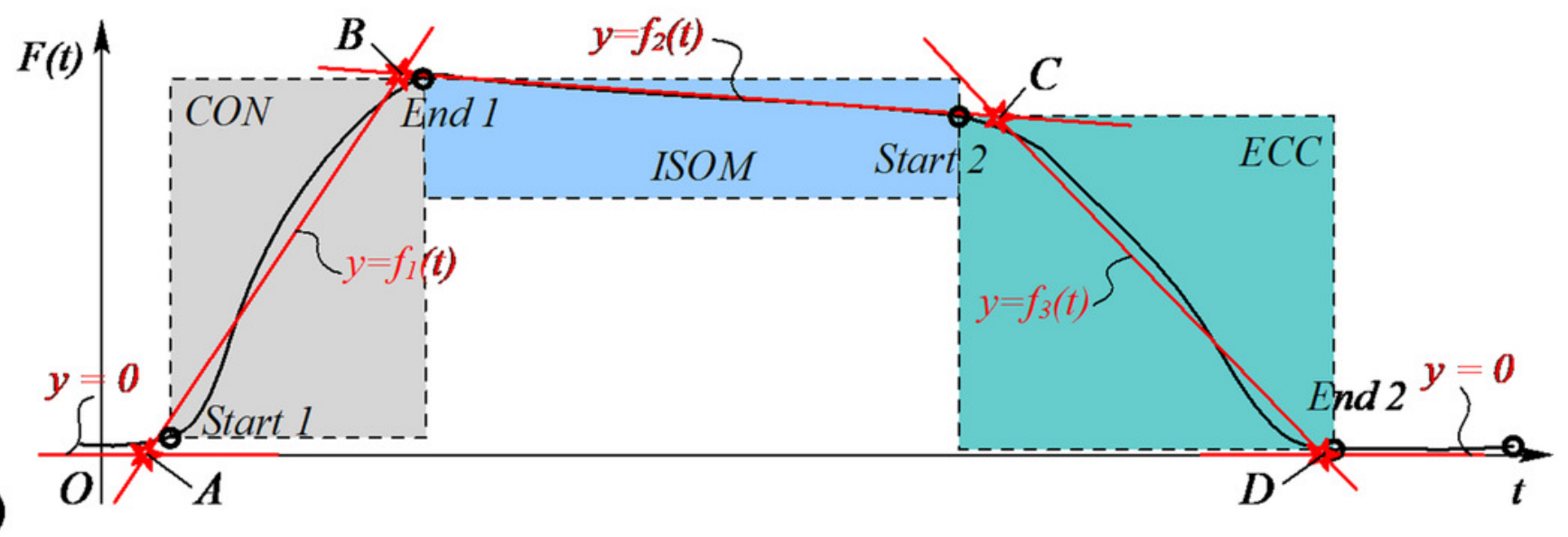


Figure 8

Average force distribution and regression trend line of a male subject.

Initial force (F0) and $\mathrm{Pl}(\mathrm{x}, \mathrm{y})$ are the mean value of the first and last three data during the 5minute ISC. P2 $(x, y)$ is the mean value of the first three data during the 2-minute ISC.

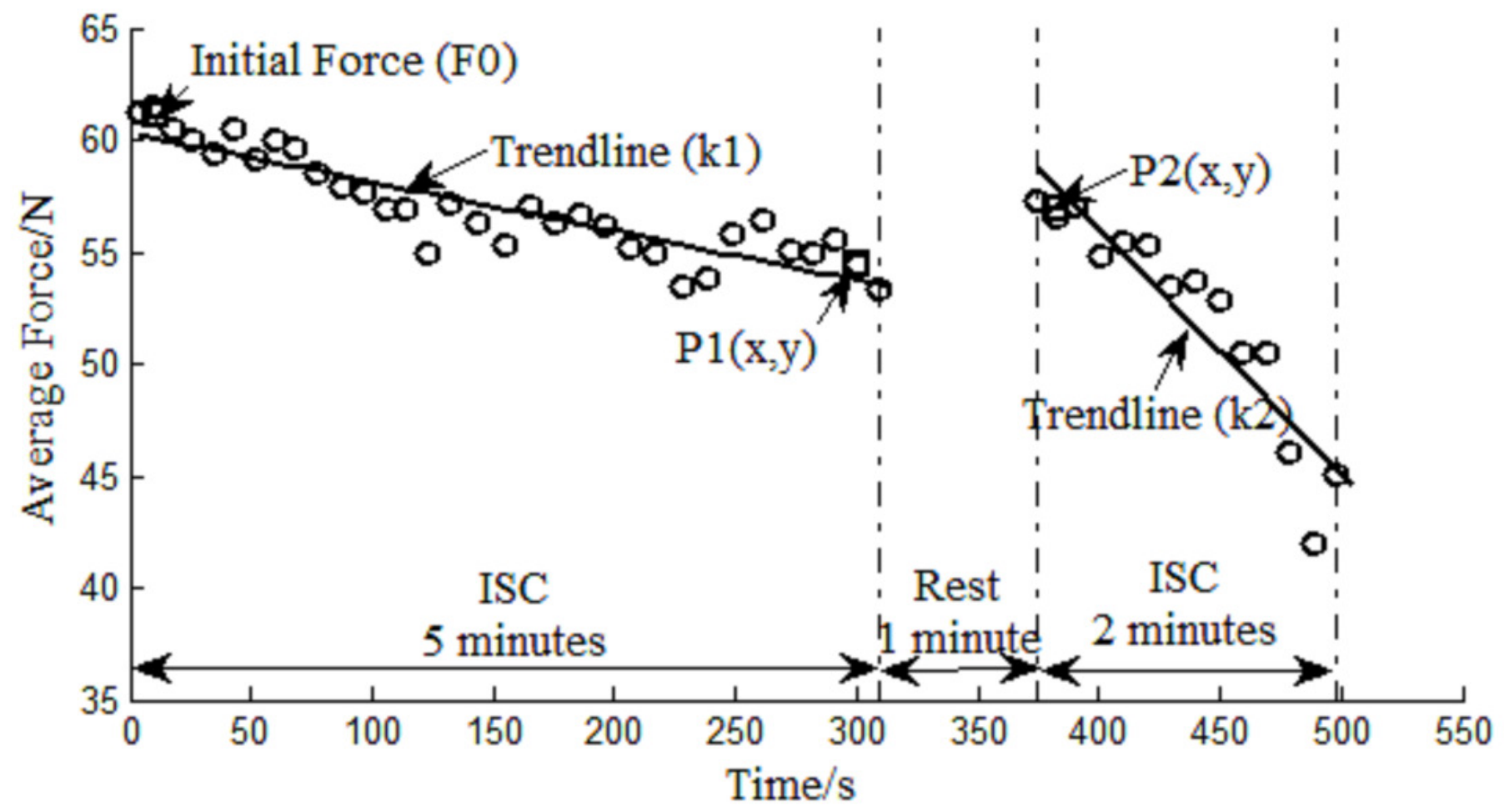




\section{Figure 9}

Schematic of the experimental procedure.

IRM is the one-repetition maximum test, MVC is the maximum voluntary contraction, and ISC is the intermittent submaximal contraction. Each black bar represents 1 repetition, which incorporates the CON, ISOM, and ECC contractions.

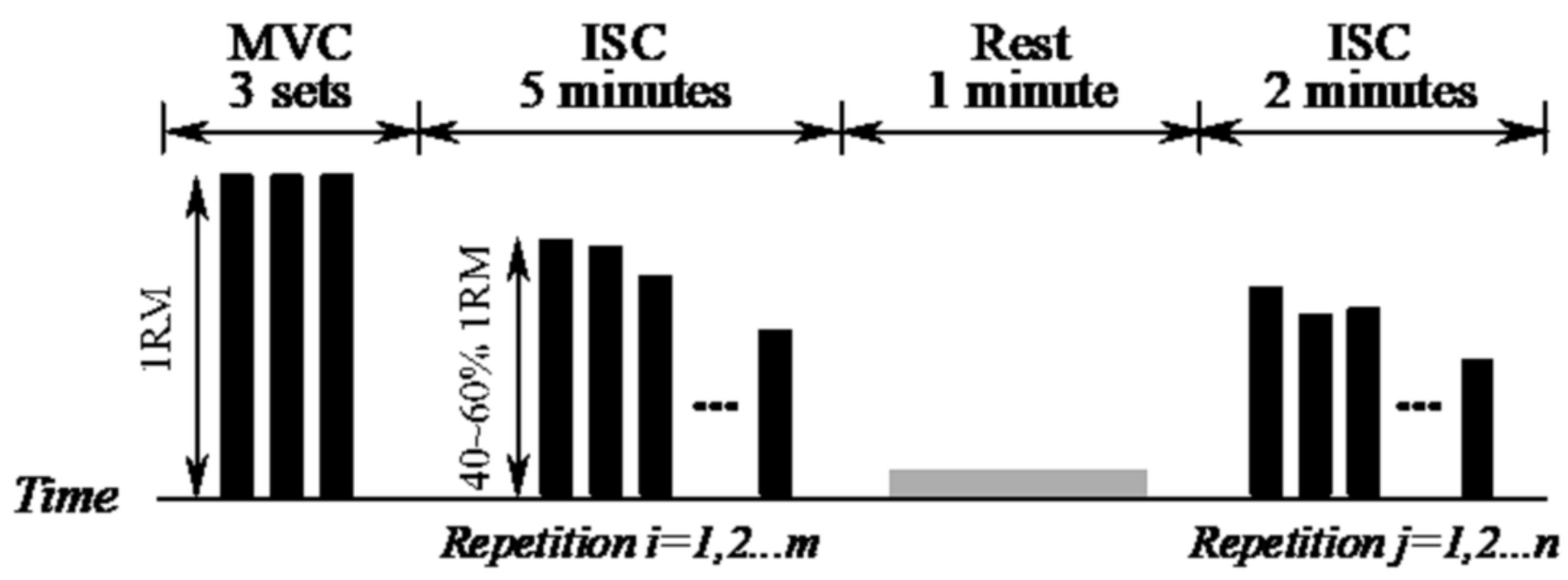


Figure 10

Representative data of a male subject.

The light blue curve represents data during the 5-minute ISC and the dark blue curve represents data during the 2 -minute ISC.

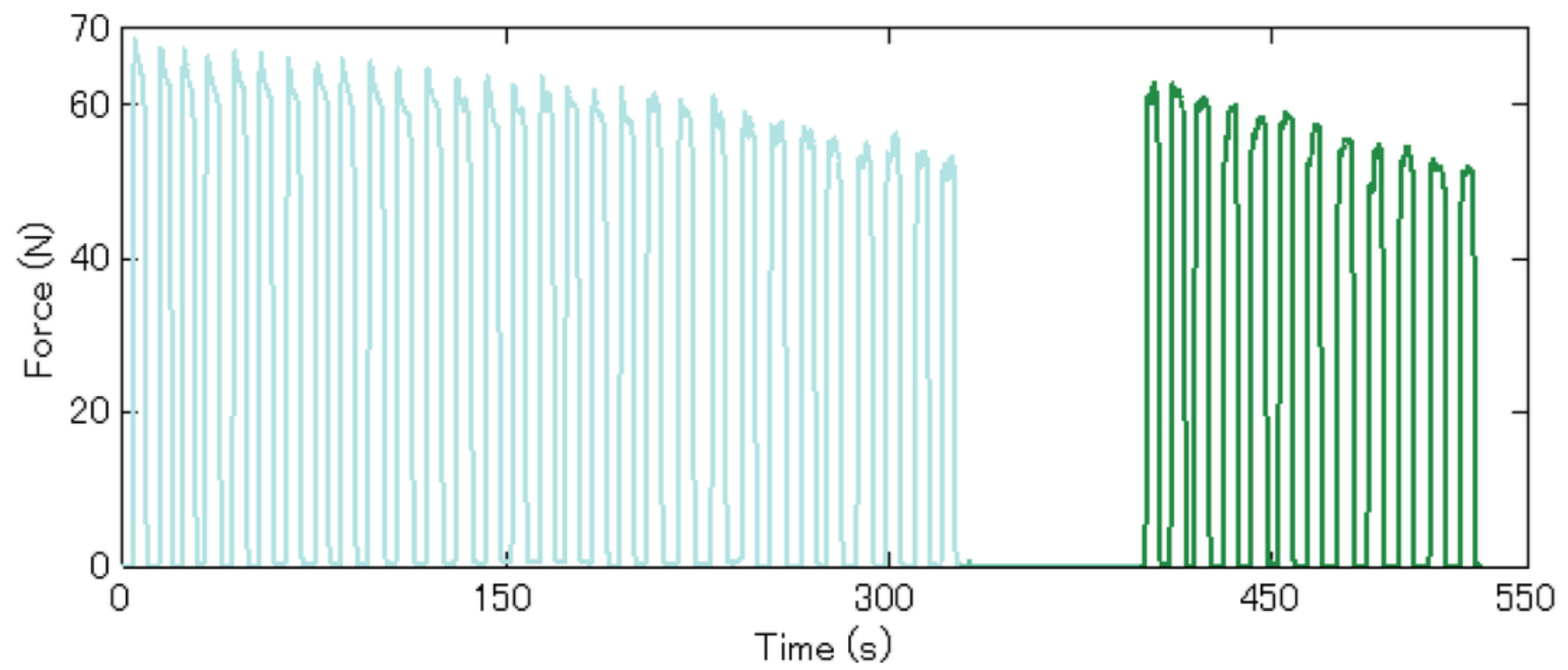


Figure 11

Normalized average force distribution and trend lines for all participants.

(A) Data of subject S01. (B) Data of subject S02. (C) Data of subject S03. (D) Data of subject S04. (E) Data of subject S05. (F) Data of subject S06.

(A): S01

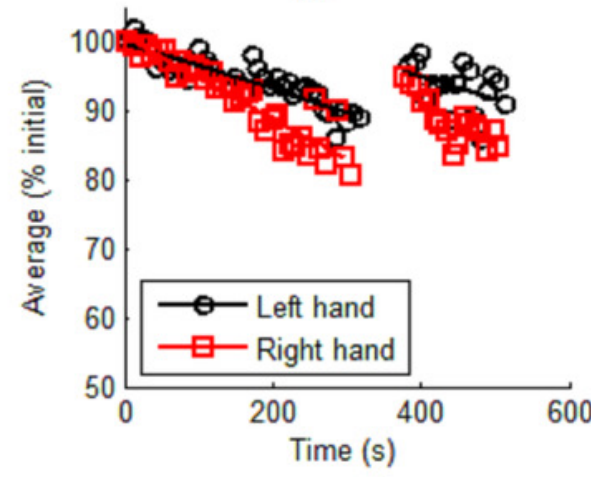

(D): S04

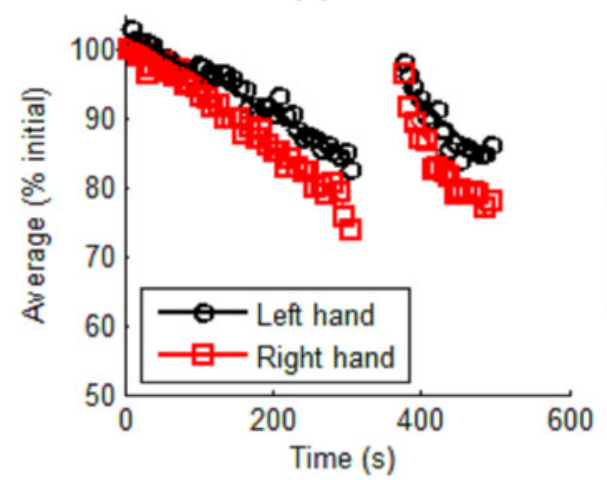

(B): S02

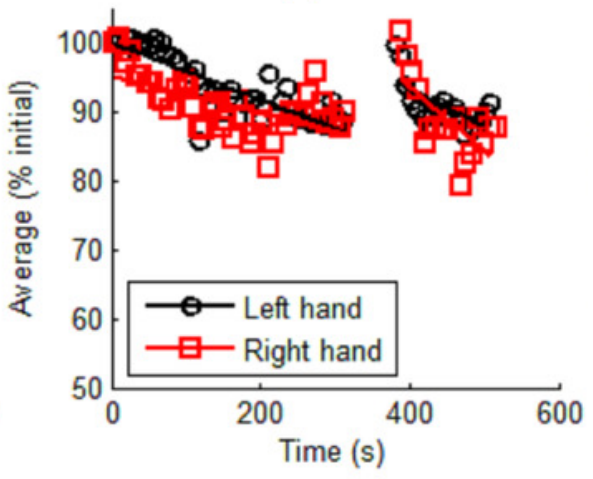

(E): S05

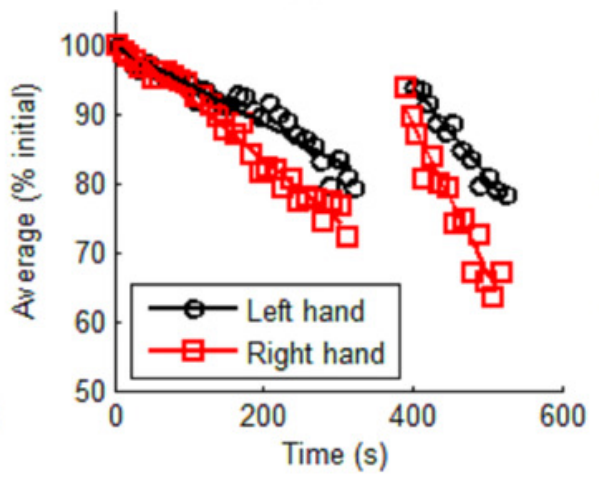

(C): S03

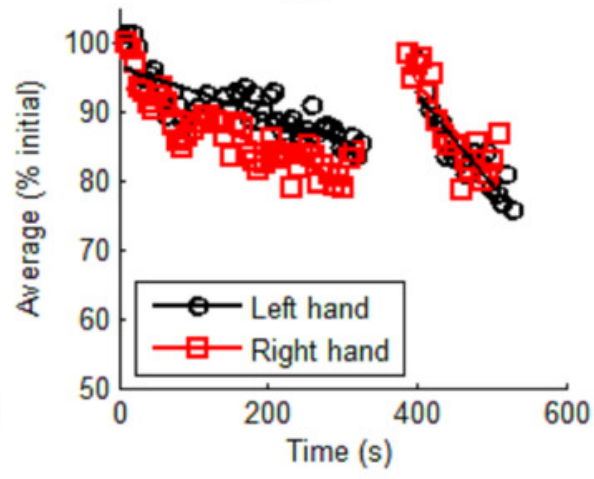

(F): S06

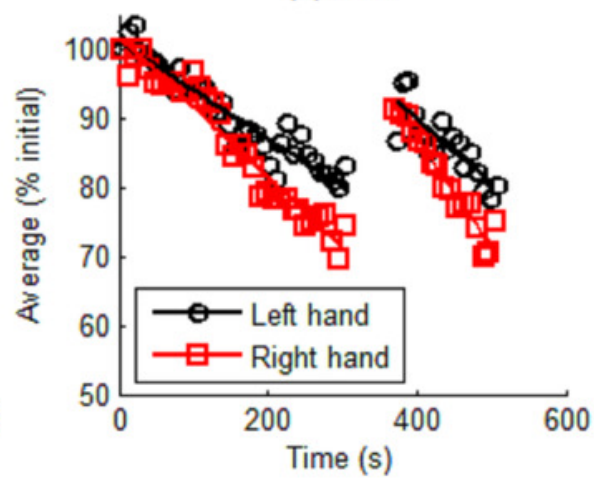


Figure 12

Four representative examples of segmentation results.

Red asterisks denote intersections and red circles denote segmentation points. The trendlines are denoted by the red lines. (A) Representative example of subject S01. (B) Representative example of subject S01. (C) Representative example of subject S01. (D) Representative example of subject S01.

(A)

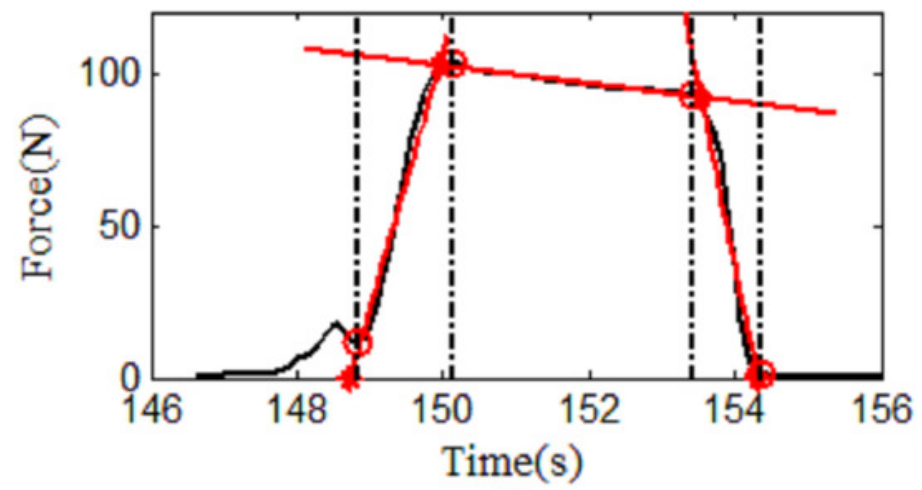

(C)

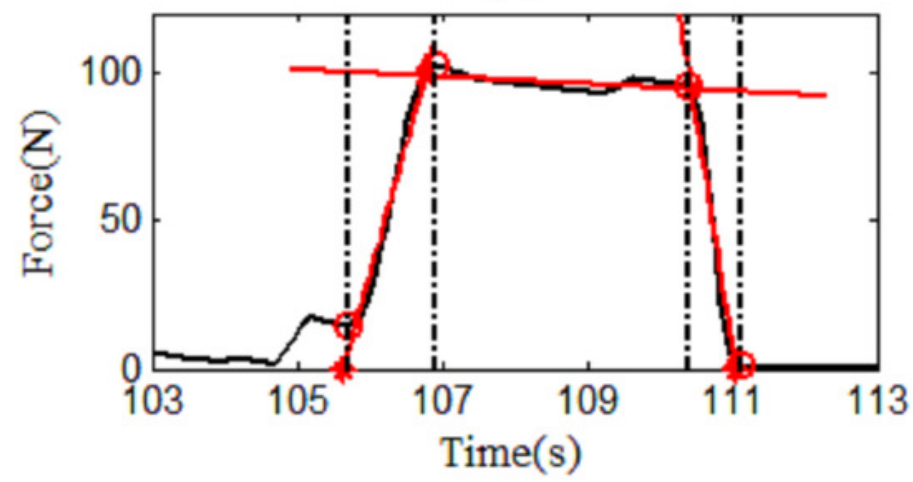

(B)

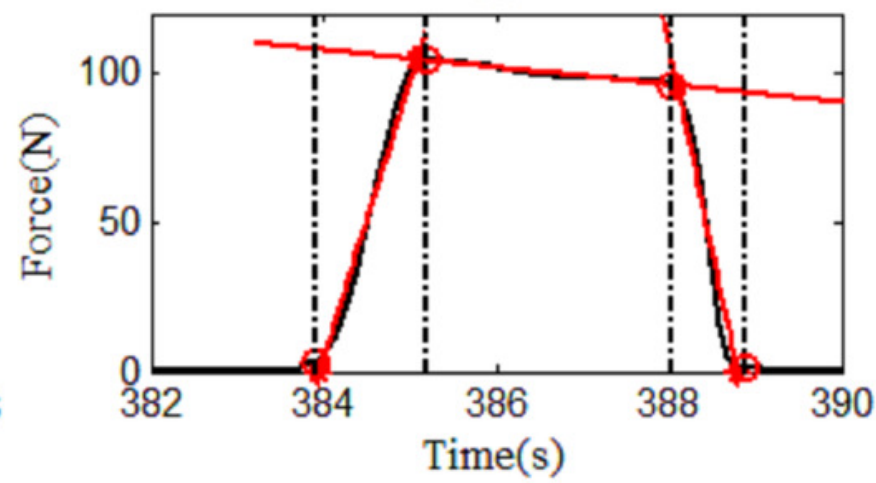

(D)

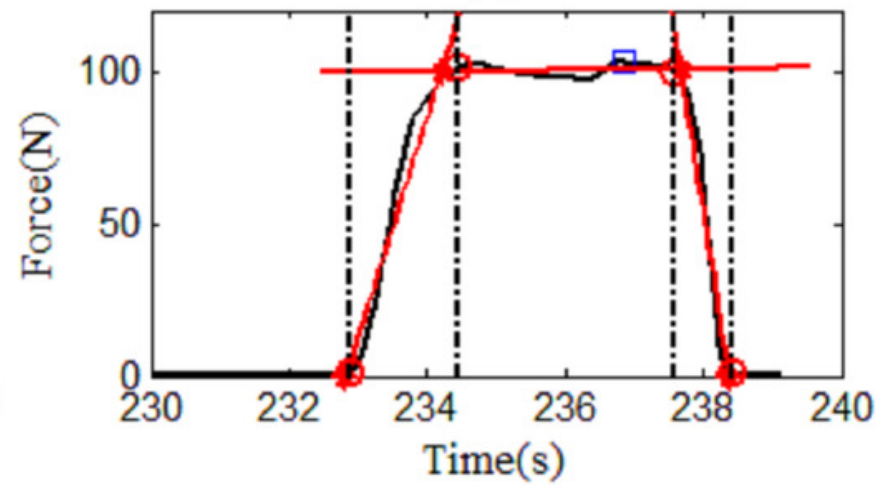


Figure 13

TUT and contraction segments comparisons for one participant at different phases.

The results show good TUT agreement and contraction segment agreement between the proposed algorithm and the assessor. (A) TUT during CON phase. (B)TUT during ISOM phase. (C)TUT during ECC phase. (D)The total TUT.
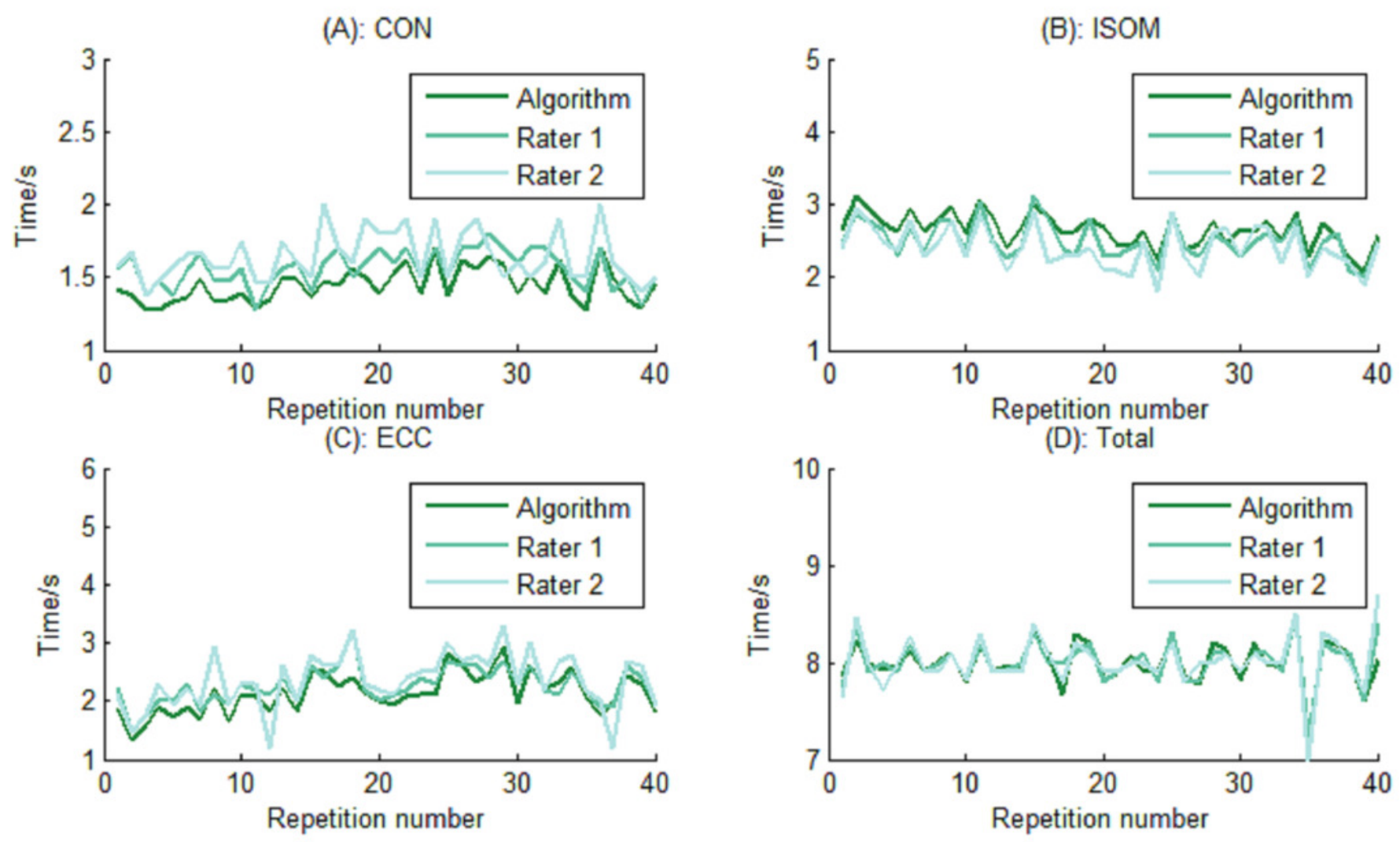
Table $\mathbf{1}$ (on next page)

Demographic data of the study participants. 
1

\begin{tabular}{|c|c|c|c|c|}
\hline \multicolumn{2}{|c|}{ Subject No. } & Age (Year) & Weight $(\mathrm{Kg})$ & Height $(\mathrm{cm})$ \\
\hline \multirow{3}{*}{ 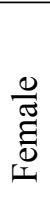 } & S01 & 34 & 50 & 170 \\
\hline & S02 & 24 & 57 & 159 \\
\hline & S03 & 27 & 48 & 160 \\
\hline \multirow{3}{*}{$\sum_{i}^{\frac{0}{\pi}}$} & S04 & 28 & 65 & 178 \\
\hline & S05 & 22 & 62 & 182 \\
\hline & S06 & 29 & 80 & 177 \\
\hline
\end{tabular}

2 


\section{Table 2 (on next page)}

The 1RM initial force combination of Thera-Bands and repetitions in the experiment.

The $2 \mathrm{R}$ means subject used 2 Red Thera-bands in exercise. 


\begin{tabular}{|c|c|c|c|c|c|c|c|}
\hline \multirow{2}{*}{\multicolumn{2}{|c|}{$\begin{array}{c}\text { Subject } \\
\text { No. }\end{array}$}} & \multirow{3}{*}{$\begin{array}{l}\text { Hand } \\
\text { Left }\end{array}$} & \multirow{3}{*}{$\begin{array}{l}1 \mathrm{RM} \\
(\mathrm{N}) \\
87.8\end{array}$} & \multirow{3}{*}{$\begin{array}{c}\text { Initial } \\
F_{\text {ave }}(\mathrm{N}) \\
45.1\end{array}$} & \multirow{3}{*}{$\begin{array}{c}\begin{array}{c}\text { Combination of } \\
\text { Thera-Bands }\end{array} \\
2 \mathrm{R}\end{array}$} & \multicolumn{2}{|c|}{ Repetitions } \\
\hline & & & & & & $m$ & $n$ \\
\hline \multirow{6}{*}{ 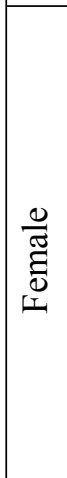 } & \multirow{2}{*}{ S01 } & & & & & 40 & 17 \\
\hline & & Right & 91.6 & 58.4 & $1 G+1 R$ & 37 & 20 \\
\hline & \multirow{2}{*}{ S02 } & Left & 67.1 & 42.0 & $1 \mathrm{R}+1 \mathrm{Y}$ & 39 & 18 \\
\hline & & Right & 59.5 & 44.7 & $1 \mathrm{R}+1 \mathrm{Y}$ & 41 & 16 \\
\hline & \multirow{2}{*}{$\mathrm{S} 03$} & Left & 108.6 & 43.0 & $1 \mathrm{G}+1 \mathrm{R}$ & 49 & 19 \\
\hline & & Right & 111.2 & 47.2 & $1 G+1 R$ & 45 & 23 \\
\hline \multirow{6}{*}{$\frac{0}{\pi^{\pi}}$} & \multirow{2}{*}{ S04 } & Left & 152.5 & 82.5 & $3 R+1 Y$ & 41 & 16 \\
\hline & & Right & 163.2 & 91.3 & $3 \mathrm{R}+1 \mathrm{Y}$ & 40 & 17 \\
\hline & \multirow{2}{*}{ S05 } & Left & 132.2 & 65.3 & $2 \mathrm{R}+1 \mathrm{Y}$ & 30 & 12 \\
\hline & & Right & 140.8 & 73.4 & $3 \mathrm{R}$ & 32 & 10 \\
\hline & \multirow{2}{*}{ S06 } & Left & 193.6 & 112.8 & $4 G+1 R$ & 37 & 17 \\
\hline & & Right & 189.6 & 120.6 & $4 G+1 R$ & 35 & 19 \\
\hline
\end{tabular}




\section{Table 3 (on next page)}

Proportion of contraction-phase specific TUT and the total TUT before and after rest.

Total TUT are the sum of TUT during CON, ISOM and ECC contraction. L denotes left hand and $\mathrm{R}$ denotes right hand. Data is expressed in percentages* and seconds**. 


\begin{tabular}{|c|c|c|c|c|c|c|c|c|c|c|c|}
\hline \multirow{2}{*}{\multicolumn{2}{|c|}{$\begin{array}{c}\text { Subject } \\
\text { No. }\end{array}$}} & \multicolumn{5}{|c|}{ Before rest } & \multicolumn{5}{|c|}{ After rest } \\
\hline & & $\mathrm{CON}^{*}$ & ISOM* & ECC* & Rest* & Total** & $\mathrm{CON}^{*}$ & ISOM* & $\mathrm{ECC}^{*}$ & Rest* & Total** \\
\hline \multirow{2}{*}{ S01 } & $\mathrm{L}$ & 18.1 & 33.2 & 26.7 & 22.0 & 249.6 & 20.1 & 32.1 & 23.3 & 24.4 & 102.1 \\
\hline & $\mathrm{R}$ & 18.3 & 33.3 & 22.8 & 25.6 & 229.4 & 17.9 & 34.3 & 26.0 & 21.7 & 105.5 \\
\hline \multirow{2}{*}{ S02 } & $\mathrm{L}$ & 11.5 & 49.9 & 8.0 & 30.7 & 216.7 & 10 & 52 & 7.6 & 30.4 & 93.9 \\
\hline & $\mathrm{R}$ & 11.3 & 44.3 & 8.3 & 36.2 & 203 & 10.4 & 41.1 & 8.5 & 40.0 & 81.5 \\
\hline \multirow{2}{*}{ S03 } & $\mathrm{L}$ & 13.4 & 67.7 & 10.3 & 8.6 & 297.3 & 14.2 & 67.2 & 9.6 & 9.0 & 119.1 \\
\hline & $\mathrm{R}$ & 14.1 & 68.5 & 11.3 & 6.1 & 295.6 & 13.3 & 67.1 & 12.3 & 7.4 & 120.7 \\
\hline \multirow{2}{*}{ S04 } & $\mathrm{L}$ & 10.3 & 50.7 & 10.7 & 28.3 & 223.2 & 9.3 & 49.5 & 9.2 & 31.9 & 85.5 \\
\hline & $\mathrm{R}$ & 11.6 & 50.3 & 12.4 & 25.7 & 228.8 & 10.6 & 48.4 & 10.5 & 30.4 & 87.1 \\
\hline \multirow{2}{*}{ S05 } & $\mathrm{L}$ & 10.8 & 37.9 & 8.4 & 42.9 & 179 & 12.3 & 37.6 & 9.4 & 40.8 & 78.8 \\
\hline & $\mathrm{R}$ & 10.9 & 35.4 & 9.6 & 44.1 & 171.5 & 11.5 & 38.9 & 10.8 & 38.8 & 69.2 \\
\hline \multirow{2}{*}{ S06 } & $\mathrm{L}$ & 8.4 & 45.7 & 11.6 & 34.4 & 203 & 9.4 & 44.6 & 9.6 & 36.4 & 92 \\
\hline & $\mathrm{R}$ & 10.1 & 44.7 & 7.6 & 37.7 & 191.7 & 11.2 & 43.7 & 8.5 & 36.6 & 89.8 \\
\hline
\end{tabular}

2 


\section{Table 4(on next page)}

Statistics of the decreasing slope of average force before and after rest.

The smaller the slope, the faster the average force drops. Student test was performed to examine the decreasing slope difference between left and right hands. Data is expressed as mean \pm standard deviation*. $p>0.05^{* *}$. 


\begin{tabular}{|c|c|c|}
\hline \multirow{2}{*}{} & \multicolumn{2}{|c|}{ Decreasing slope of average force } \\
\cline { 2 - 3 } & Before rest (k1) & After rest (k2) \\
\hline Female & $-0.0407 \pm 0.0115^{*}$ & $-0.0860 \pm 0.0416^{*}$ \\
\hline Male & $-0.0758 \pm 0.0176^{*}$ & $-0.140 \pm 0.0425^{*}$ \\
\hline Overall & $-0.0582 \pm 0.0231^{*}$ & $-0.113 \pm 0.0492^{*}$ \\
\hline T-test & $\mathrm{p}=0.2^{* *}$ & $\mathrm{p}=0.14^{* *}$ \\
\hline
\end{tabular}

2 


\section{Table 5 (on next page)}

Statistics of 1 MinRec in left and right hand.

Data are expressed as mean \pm standard deviation; Student test was performed to examine the 1 MinRec difference between left and right hands. $p<0.05 *$. 


\begin{tabular}{|c|c|}
\hline & 1MinRec (\%Initial) \\
\hline Left hand & $9.968 \pm 1.889$ \\
\hline Right hand & $13.738 \pm 3.149$ \\
\hline Overall & $11.853 \pm 3.163$ \\
\hline T-test & $p=0.03 *$ \\
\hline
\end{tabular}

2 